\title{
Comparative transcriptome and metabolome analyses provide new insights into the molecular mechanisms underlying taproot thickening in Panax notoginseng
}

Xue-Jiao Li ${ }^{1,2+}$, Jian-Li Yang ${ }^{3 \dagger}$, Bing Hao ${ }^{1 \dagger}$, Ying-Chun Lu', Zhi-Long Qian ${ }^{1}$, Ying Li', Shuang Ye ${ }^{1}$, Jun-Rong Tang ${ }^{1}$, Mo Chen ${ }^{1}$, Guang-Qiang Long ${ }^{1}$, Yan Zhao ${ }^{1}$, Guang-Hui Zhang ${ }^{1}$, Jun-Wen Chen ${ }^{1}$, Wei Fan ${ }^{1 *}$ and Sheng-Chao Yang ${ }^{1 *}$

\begin{abstract}
Background: Taproot thickening is a complex biological process that is dependent on the coordinated expression of genes controlled by both environmental and developmental factors. Panax notoginseng is an important Chinese medicinal herb that is characterized by an enlarged taproot as the main organ of saponin accumulation. However, the molecular mechanisms of taproot enlargement are poorly understood.

Results: A total of 29,957 differentially expressed genes (DEGs) were identified during the thickening process in the taproots of P. notoginseng. Gene Ontology and Kyoto Encyclopedia of Genes and Genomes pathway enrichment revealed that DEGs associated with "plant hormone signal transduction," "starch and sucrose metabolism," and "phenylpropanoid biosynthesis" were predominantly enriched. Further analysis identified some critical genes (e.g., RNase-like major storage protein, DA1-related protein, and Starch branching enzyme I) and metabolites (e.g., sucrose, glucose, fructose, malate, and arginine) that potentially control taproot thickening. Several aspects including hormone crosstalk, transcriptional regulation, homeostatic regulation between sugar and starch, and cell wall metabolism, were identified as important for the thickening process in the taproot of P. notoginseng.

Conclusion: The results provide a molecular regulatory network of taproot thickening in P. notoginseng and facilitate the further characterization of the genes responsible for taproot formation in root medicinal plants or crops.
\end{abstract}

Keywords: Panax notoginseng, Taproot thickening, Signal transduction, Metabolic regulation

\section{Background}

Root/stem thickening presents as an enlarged structure in some plant species where it is used as a storage organ for nutrients, thus providing food for humans and animals. Root/stem thickening is mainly regulated by the vascular system as a consequence of the interaction between the primary and secondary cambium. The

\footnotetext{
*Correspondence: fanwei1128@aliyun.com; shengchaoyang@163.com ${ }^{+}$Xue-Jiao Li, Jian-Li Yang and Bing Hao contributed equally to this work. ${ }^{1}$ State Key Laboratory of Conservation and Utilization of Bio-resources in Yunnan, The Key Laboratory of Medicinal Plant Biology of Yunnan Province, National\& Local Joint Engineering Research Center on Germplasm Innovation \& Utilization of Chinese Medicinal Materials in Southwest China, Yunnan Agricultural University, Kunming 650201, China

Full list of author information is available at the end of the article
}

primary cambium determines the primordium formation of the root/stem, while the secondary cambium affects root/stem thickening by increasing cell number and volume [1]. Studies have shown that signal regulation and metabolic change are the main endogenous factors during root/stem thickening, while environmental factors such as soil temperature, humidity, light, photoperiod, carbon dioxide, and drought also affect this process [2-6]. A comprehensive understanding of the molecular mechanisms regulating various aspects of root/stem thickening is required to facilitate the development of new plant varieties.

"Omics" technologies have contributed to our understanding of the molecular mechanisms underlying root/ 
stem thickening. The fleshy taproot of radish (Raphanus sativus) is an important storage organ that determines radish yield and quality. Genome sequencing and transcriptome analysis indicated that the genes related to carbohydrate metabolism were prominently activated during taproot thickening [7, 8]. Proteomic analysis identified that cell division cycle 5-like protein (CDC5), expansin B1 (EXPB1), and xyloglucan endotransglucosylase/hydrolase protein 24 (XTH24) were responsible for the cell division and expansion of taproot thickening in radish [9]. Further high-throughput sequencing of small RNA indicated that microRNAs were also specific at different developmental stages, and their targets encoding transcription factors (TFs) and other functional proteins, including nuclear TF $\mathrm{Y}$ subunit A-2 protein (NF-YA2), IAA-amino acid hydrolase ILR1 gene (ILR1), basic helixloop-helix TF bHLH74 (bHLH74), CLAVATA3/ESR-related 41 (CLE41), XTH16 and EXPA9 were involved in radish taproot thickening [10]. In onion (Allium cepa), the rapid swelling of the bulb results in a significantly decreased sucrose content at an early stage of development, whereas fructose and glucose contents increase significantly at the mature stage, suggesting that sucrose metabolism plays an important role in onion bulb formation [11]. Additionally, Wang [12] reported that root tuberization in cassava (Manihot esculenta) was correlated with the significant up-regulated expression of carbohydrate metabolic enzymes. Of these, the 14-3-3 proteins and their binding enzymes might play important roles in carbohydrate metabolism and starch accumulation during cassava root tuberization. Similar results were also reported in potato (Solanum tuberosum) [13]. These results indicated that root/stem thickening is a complex trait that is controlled by multiple biological processes/pathways. However, these studies mainly focused on crops, and there is still a lack of understanding of the root/stem thickening process in other plants, particularly medicinal plants.

The enlarged root, stem and rhizome in medicinal plants are important medicinal organs that accumulate numerous active ingredients and account for one-third of the species recorded in the Chinese Pharmacopoeia. At present, many studies have aimed to elucidate the relationship between the development of enlarged tissues and the accumulation of secondary metabolites. For example, comparative transcriptome studies between tuberous roots and shoots in Aconitum heterophyllum showed that 3-hydroxy-3-methylglutaryl-CoAreductase (HMGR), mevalonate kinase (MVK), mevalonate diphosphate decarboxylase (MVDD), and 1-hydroxy-2-methyl-2(E)-butenyl 4-diphosphate synthase (HDS) were required for aconite alkaloid biosynthesis, while genes encoding TFs, including bHLH, MYB basic leucine zipper (bZIP), and ATP-binding cassette $(\mathrm{ABC})$ transporter, were implicated in tuberous root development [14]. In Ophiopogon japonicus, genes related to flavonoid and saponin synthesis were negatively regulated with tuberous root development, while genes related to polysaccharide synthesis were positively regulated in the early stage and negatively regulated in the later stage [15]. However, these studies lack a systematic investigation of the enlargement of these tissues.

Panax notoginseng (Burk) F.H.Chen is one of the most famous Chinese traditional medicinal plants belonging to the Panax genus in the Araliaceae family [16]. Triterpenoid saponins are the main active components that are widely used in the treatment of stroke injury, coronary heart disease, angina pectoris, cerebrovascular sequelae, hypertension and other diseases in $P$. notoginseng [17-19]. Currently, P. notoginseng is the main raw material of more than 400 Chinese patented medicines with an annual demand of 8 million kilograms and a total output value of more than 70 billion $\operatorname{RMB}[20,21]$. Taproot thickening in $P$. notoginseng is responsible for saponin accumulation, thus directly affecting its quality and yield. Traditionally, the quality of $P$. notoginseng has mainly been evaluated according to the appearance and size of the taproot, while the harvest period is primarily determined based on the taproot yield. However, there is a lack of understanding of the molecular mechanisms regulating taproot morphogenesis in $P$. notoginseng. In this study, the expression profiles of key stages during taproot thickening were constructed using one-year-old $P$. notoginseng plants. Further integrated transcriptomic and metabolomic analysis revealed the regulatory networks and critical genes potentially controlling taproot thickening. These results should serve as an important public reference platform for identifying the functions of candidate genes, thus facilitating the dissection of the molecular mechanisms underlying taproot development in P. notoginseng.

\section{Results \\ Morphological changes during P. notoginseng taproot thickening}

We measured the dynamic growth indexes of the taproots of one-year-old $P$. notoginseng plants monthly. The results showed that the seed root germinated in March, while thickening took place from May to July and stabilized from August to November (Fig. 1a). However, the taproot elongation was not significant until July in comparison with March (Fig. 1b). Both the fresh and dry weight of the taproot increased with the growth period, but there was no significant difference from August to November (Fig. 1c). Similarly, the total saponins also increased with the growth month, but there was no significant difference from September to November (Fig. 1d). 
A

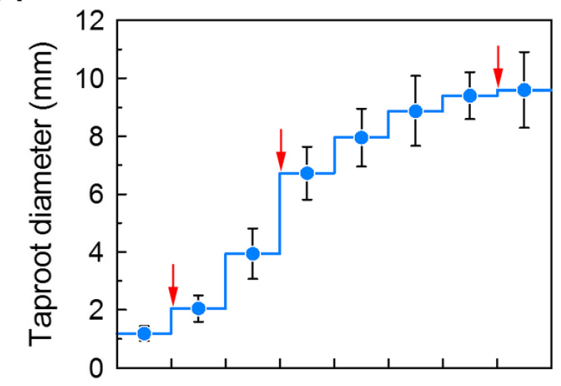

C

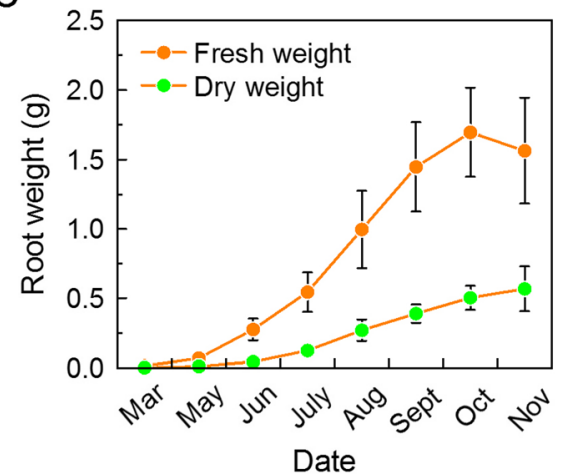

B

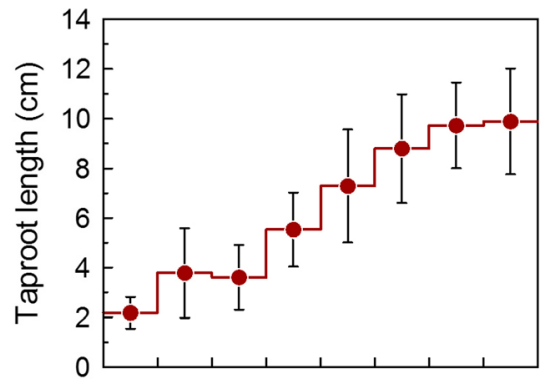

D

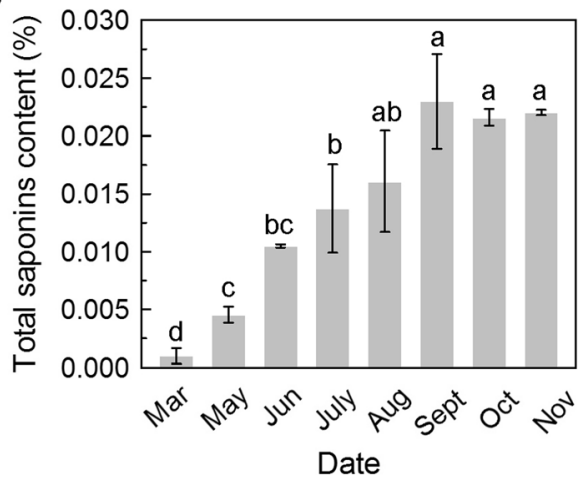

Fig. 1 Determination of growth indices of $P$. notoginseng taproots at different developmental stages. a Taproot diameter. b Taproot length. c Taproot weight. d Total saponins content. Data are expressed as means \pm SD $(n=30)$. Columns with different letters are significantly different at $P<0.05$

This suggested that fresh weight is indicative of saponin content during the first year of $P$. notoginseng growth.

Taproot thickening is associated with secondary meristem activity. We observed the anatomical structure of the taproot during different months of growth. The results showed that the vascular bundles were not well developed in the seed root stage in March (Fig. 2a). The division of the secondary cambium initiated taproot thickening in May (Fig. 2b, c) and divided continuously in July, which produced secondary phloem cells outward and secondary xylem cells inward that promoted rapid thickening of the taproot (Fig. 2d, e). From October, the thickening rate of the taproot slowed down and tended to stabilize (Fig. 2f, g). These results allowed us to identify four key stages associated with taproot thickening in $P$. notoginseng: seed root stage (March), initial thickening stage (May), rapid thickening stage (July) and stable thickening stage (November).

\section{RNA sequencing analysis of the developing taproot of $P$. notoginseng}

To explore the molecular basis of the morphological changes during taproot thickening, RNA sequencing (RNA-Seq) analysis of the taproots at four different stages was performed. Principal component analysis (PCA) revealed that the 12 samples could be clearly assigned to four groups. There was a significant difference between March and May, while July and November clustered together, suggesting that the overall transcriptomic profiles between the seed root and initial thickening stages were distinct, while those of the rapid and stable thickening stages were similar (Fig. 3a). In order to obtain reliable gene expression profiles, reads with $\log _{2} \mid$ Fold Change $\mid>1$ and RPKM values $>10$ were selected to annotate the differentially expressed genes (DEGs). A total of 9079 upregulated DEGs and 20,878 down-regulated DEGs were derived from the comparison between the different stages. The number of down-regulated DEGs was higher than that of the up-regulated genes in March, May and July, whereas the opposite occurred in November (Fig. 3b). The up- or down-regulated DEGs in the different taproot thickening stages are listed in Additional file 1: Table S1. A Venn diagram of the DEGs showed that none were upregulated consistently in May, July and November vs. March, while 12 genes were up-regulated consistently in May and July vs. March (Fig. 3c). By contrast, four genes were down-regulated consistently in May, July, and November vs. March, while 43 genes were down-regulated in May and July vs. March (Fig. 3d). As initial and rapid thickening are the key stages for taproot development, the consistently up-regulated or down-regulated genes in May and July vs. March play an important role in taproot development in $P$. notoginseng. In fact, the taproot almost stopped thickening in September (Fig. 1a). Therefore, the change in gene expression profile was not significant in the stable thickening stage, as reflected by the number of 


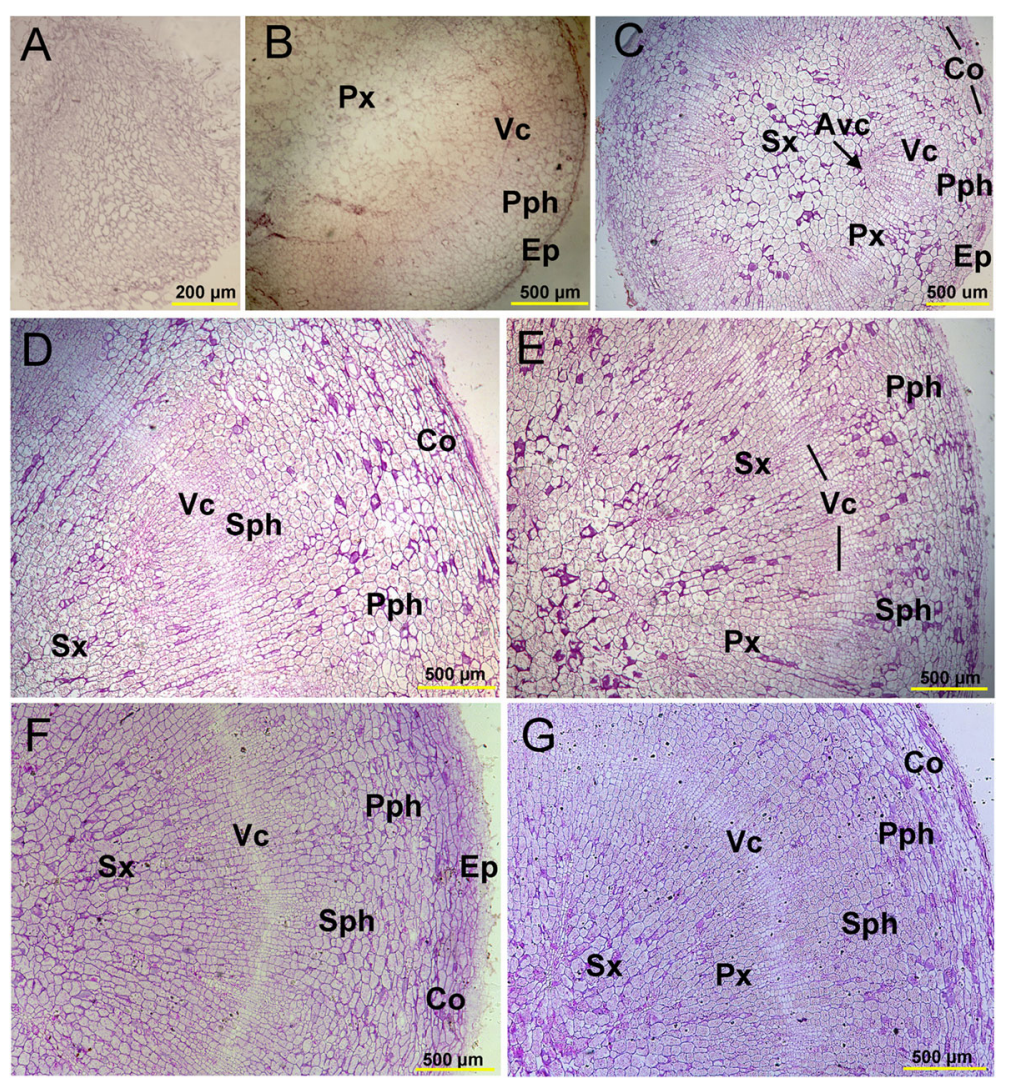

Fig. 2 Anatomical structure of $P$. notoginseng taproots at different developmental stages. a March. b-e May to August. f, g October-November. Avc, accessory cambium. Co, cortex. Ep, epidermis. Ppc, phloem parenchyma cell. Pph, primary phloem. Px, primary xylem. Sph, secondary phloem. Sx, secondary xylem. Vc, vascular cambium

DEGs in November vs. July (Fig. 3b). The high-quality reads obtained in this study were deposited in the NCBI SRA database (accession number: SUB5611240).

The DEGs were further categorized and characterized according to the functional category defined by the Gene Ontology (GO) and Kyoto Encyclopedia of Genes and Genomes (KEGG) pathway. GO analysis showed that the DEGs were mainly involved in "metabolic process," followed by "cell process" and "single-organism process" (Additional file 2: Figure S1). Metabolic processes analysis by KEGG showed that the DEGs were predominantly enriched in "plant hormone signal transduction pathway," "starch and sucrose metabolism pathway," and "phenylpropanoid biosynthesis pathway" (Fig. 4), suggesting that these pathways may be closely related to taproot thickening in P. notoginseng.

\section{Identifying critical genes controlling taproot development in $P$. notoginseng}

According to the Venn diagram, 12 DEGs were significantly up-regulated in the pairwise comparisons among May vs. March, July vs. March, and July vs. May. These genes were mainly related to "carbohydrate and storage metabolism," including genes encoding RNase-like major storage protein, Beta-amylase (BAMY) 5, Alpha-1,4 glucan phosphorylase L isozyme, Starch branching enzyme (SBE) I and Vacuolar protein sorting-associated protein 32 homolog 2, and "signal transduction and transcription," including genes encoding Protein phosphatase 2C 37-like, Two-component response regulator-like APRR7, and DA1-related protein 5 (Table 1). In contrast, 43 down-regulated DEGs overlapped among the abovementioned comparisons and were mainly involved in "signal transduction and transcription," "stress/defense response," and "other metabolisms," including lipid metabolism, secondary metabolism, and cell wall metabolism (Table 2). Noticeably, four genes showing high homology with Delta(12)-fatty-acid desaturase FAD2like (AtAT3G12120) were identified as consistently down-regulated during taproot thickening (Table 2). These results suggested that the genes associated with signal transduction, transcriptional regulation and metabolic pathways play diverse roles in taproot thickening in P. notoginseng. 


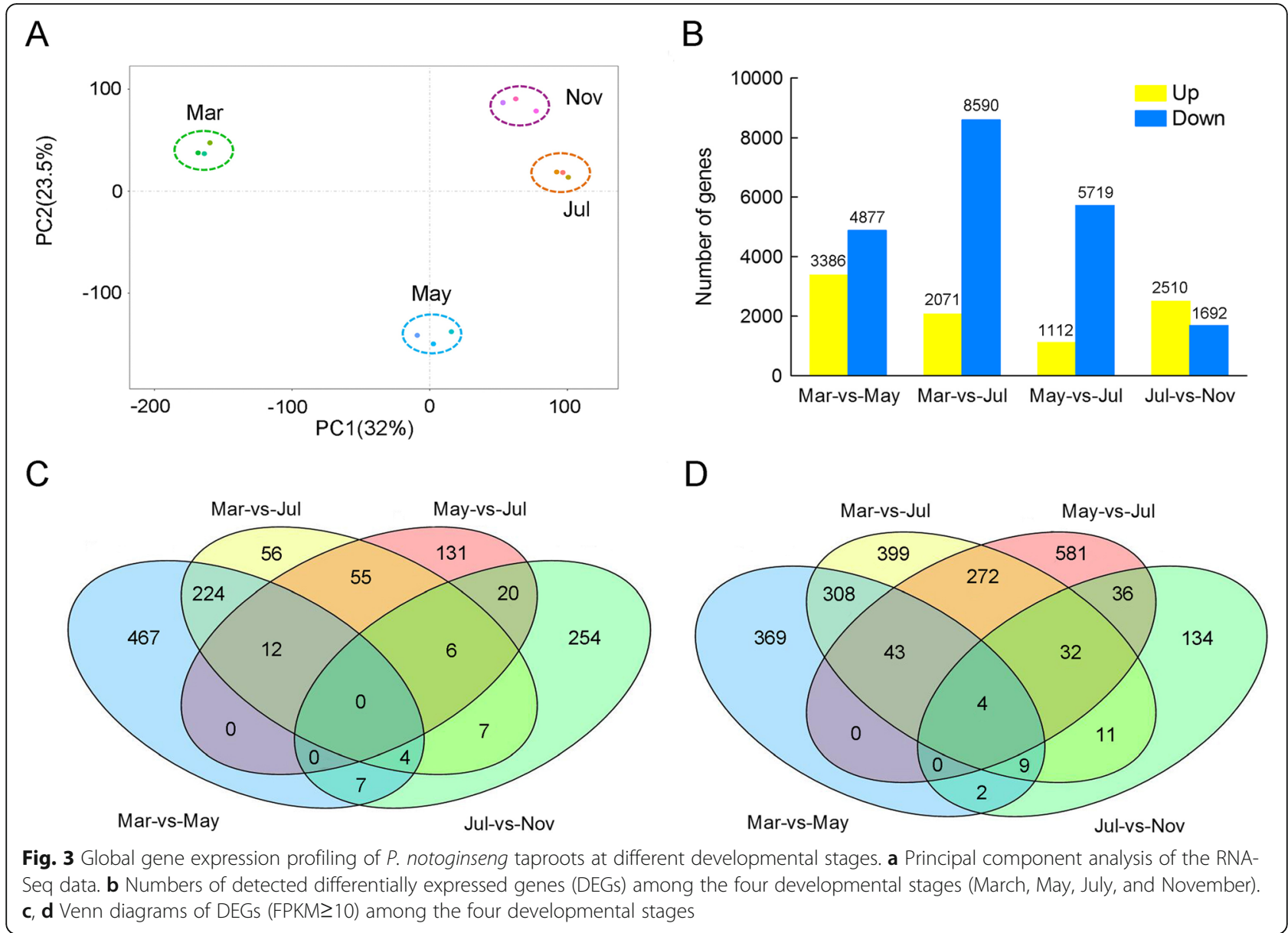

To validate the reliability of the transcriptomic data, we selected 16 DEGs that include 12 DEGs mentioned above for the quantitative real-time PCR (qRT-PCR) analysis. There was a good correlation $(r=0.83)$ between the transcriptomic data and the qRT-PCR results (Additional file 3: Figure S2). These results indicated that the transcriptomic data could reflect the transcriptional changes during the thickening process in the taproots of P. notoginseng.

\section{Differential expression of TFs}

A total of 237 DEGs were identified and assigned to 30 TF families (Additional file 4: Table S2). Among them, 97 DEGs encoding APETALA2/ethylene-responsive factor (AP2/ERF), WRKY, bHLH, NAC domain containing protein (NAC), BRI1-EMS-SUPPRESSOR 1 (BES1), Cys2His2 zinc finger protein $(\mathrm{C} 2 \mathrm{H} 2)$, and Auxin response factor (ARF), have previously been implicated in plant growth and development [22-28] (Fig. 5). APETALA2/ ethylene response factor (AP2/ERF) TF is one of the largest superfamilies in the plant kingdom, indicating that different members play a specific role in different taproot thickening stages (Fig. 5). Genes encoding the $\mathrm{C} 2 \mathrm{H} 2$ and ARF families were predominantly up-regulated in March, while genes encoding the WRKY, bHLH, NAC, and BES1 families were up-regulated mainly in March and May (Fig. 5). These results suggest that the up-regulated TF families may be involved in the early developmental regulation of P.notoginseng taproot thickening.

\section{Differential expression of hormone signaling transcripts}

The involvement of plant hormones, including indole-3acetic acid (IAA), cytokinin (CTK), gibberellin (GA), ethylene (ETH), jasmonate (JA), and brassinosteroid (BR), in the development and formation of the roots has been investigated previously $[29,30]$. Our KEGG analysis showed that "plant hormone signal transduction pathway" was predominantly enriched during taproot thickening in P. notoginseng (Fig. 4), with 93 DEGs grouped into the IAA, BR, abscisic acid (ABA), GA, ETH, JA, zeatin (ZT), salicylic acid (SA), and CTK pathways. While DEGs associated with the GA signaling pathway were up-regulated mainly in November, DEGs related to the ABA signaling pathway were significantly up- 


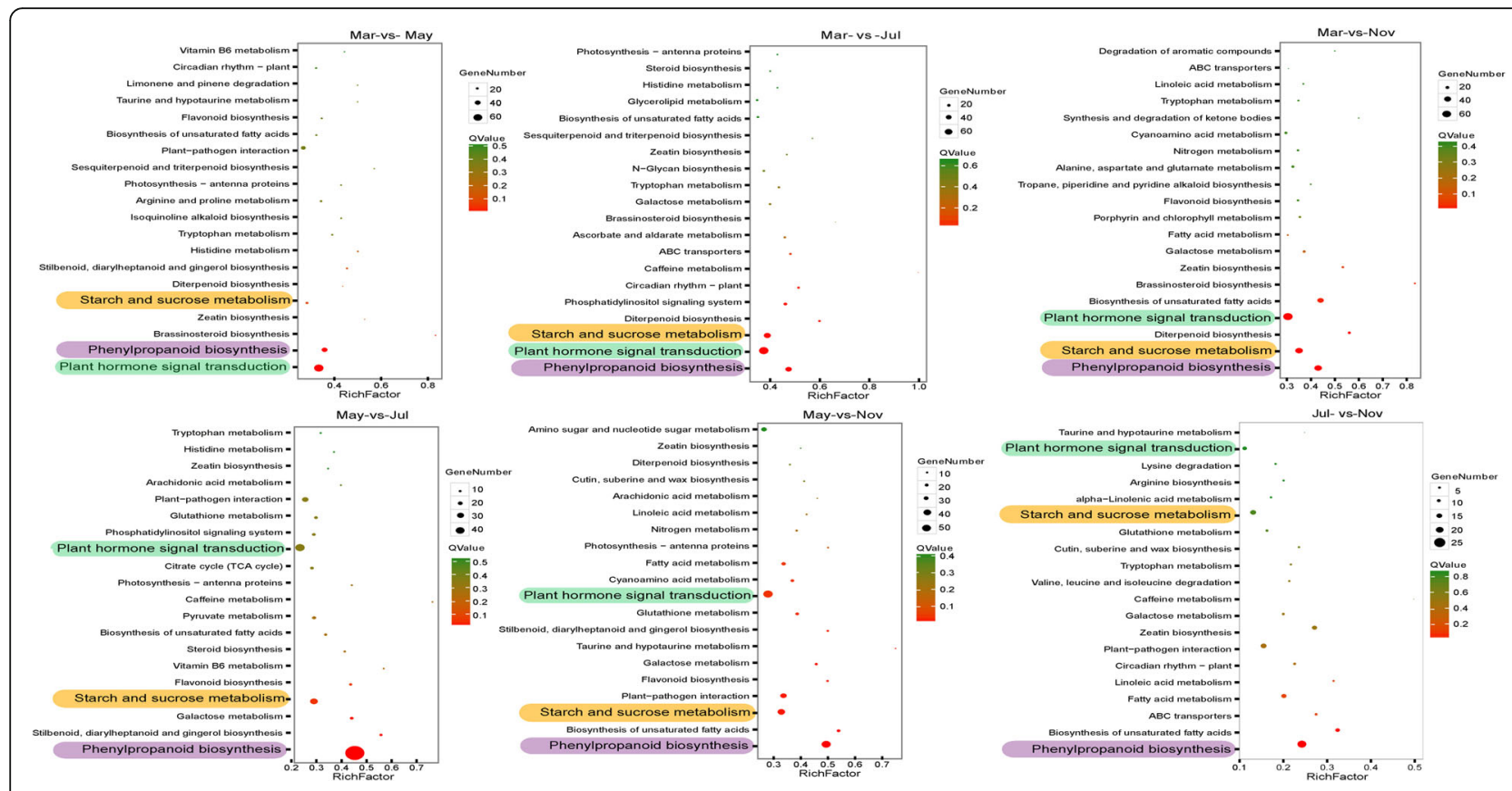

Fig. 4 Significantly enriched KEGG pathways of DEGs. Top 20 significantly enriched KEGG pathways at different developmental stages. The Y-axis on the left represents KEGG pathways, and the X-axis indicates the "enrich factor" represented by the ratio of DEGs numbers to total annotated gene numbers of each pathway. Low q-values are shown in the red circle, and high q-values are shown in the green circle. The area of a circle represents DEGs number. The KEGG pathways, associated with the starch and sucrose metabolism, phenylpropanoid biosynthesis, and plant hormone signal transduction are underlined using the orange, purple, and aqua bar, respectively

Table 1 Identification of up-regulated DEGs at initial (May) and rapid (July) thickening stages of P. notoginseng taproots

\begin{tabular}{|c|c|c|c|c|c|c|}
\hline \multirow[t]{2}{*}{ ID } & \multirow[t]{2}{*}{ Description } & \multirow[t]{2}{*}{ TAIR ID } & \multicolumn{4}{|l|}{ RPKM } \\
\hline & & & Mar & May & Jul & Nov \\
\hline \multicolumn{7}{|c|}{ Carbohydrate and storage metabolism } \\
\hline Unigene0007855 & RNase-like major storage protein & AT2G02990 & 22.3 & 3071.0 & $129,797.0$ & $44,296.7$ \\
\hline Unigene0036675 & RNase-like major storage protein & AT2G02990 & 25.6 & 5736.2 & $140,415.0$ & $48,725.4$ \\
\hline Unigene0001769 & Beta-amylase 5 & AT4G15210 & 55.9 & 3345.0 & $11,618.7$ & 4698.6 \\
\hline Unigene0035964 & Alpha-1,4 glucan phosphorylase L isozyme & AT3G29320 & 8.4 & 302.2 & 728.3 & 570.6 \\
\hline Unigene0011567 & Starch branching enzyme I & AT2G36390 & 18.4 & 259.8 & 1087.5 & 850.9 \\
\hline Unigene0036902 & Vacuolar protein sorting-associated protein 32 homolog 2 & AT4G29160 & 8.1 & 194.4 & 426.2 & 369.6 \\
\hline \multicolumn{7}{|c|}{ Signal transduction and transcription } \\
\hline Unigene0032604 & Protein phosphatase 2 C 37 -like & AT3G11410 & 11.9 & 19.1 & 53.7 & 77.2 \\
\hline Unigene0014980 & Two-component response regulator-like APRR7 & AT5G53420 & 20.5 & 39.0 & 209.9 & 90.9 \\
\hline Unigene0011239 & DA1-related protein 5 & AT5G62580 & 18.6 & 26.4 & 38.4 & 29.5 \\
\hline \multicolumn{7}{|c|}{ Secondary metabolism } \\
\hline Unigene0034150 & GBR5 & / & 208.3 & 357.5 & 810.6 & 601.6 \\
\hline Unigene0036783 & Heterodimeric geranylgeranyl pyrophosphate synthase small subunit & AT4G38460 & 23.6 & 232.5 & 1157.4 & 282.1 \\
\hline \multicolumn{7}{|l|}{ Undefined } \\
\hline Unigene0002182 & Aspartate, glycine, lysine and serine-rich protein & AT5G20190 & 29.0 & 118.0 & 207.9 & 403.3 \\
\hline
\end{tabular}


Table 2 Identification of down-regulated DEGs at initial (May) and rapid (July) thickening stages of P. notoginseng taproots

\begin{tabular}{|c|c|c|c|c|c|c|}
\hline \multirow[t]{2}{*}{ ID } & \multirow[t]{2}{*}{ Description } & \multirow[t]{2}{*}{ TAIR ID } & \multicolumn{4}{|l|}{ RPKM } \\
\hline & & & Mar & May & Jul & Nov \\
\hline \multicolumn{7}{|c|}{ Signal transduction and transcription } \\
\hline Unigene0046518 & AP2/ERF domain-containing transcription factor DREB1D & AT5G51190 & 187.6 & 52.7 & 23.5 & 23.2 \\
\hline Unigene0019102 & Ethylene-responsive transcription factor 2-like & AT4G17500 & 312.9 & 103.8 & 47.3 & 50.4 \\
\hline Unigene0036608 & Auxin-responsive protein IAA7-like & AT3G23050 & 253.7 & 94.7 & 47.3 & 30.3 \\
\hline Unigene0039183 & 60 kDa jasmonate-induced protein-like & / & 132.9 & 50.8 & 13.2 & 16.0 \\
\hline Unigene0018277 & Calcium-binding protein PBP1-like & AT5G54490 & 107.9 & 50.6 & 19.4 & 19.6 \\
\hline Unigene0017010 & Snakin-2-like & AT1G75750 & 152.6 & 60.2 & 16.9 & 9.0 \\
\hline Unigene0000195 & Organ-specific protein S2-like & / & 5774.5 & 2708.3 & 893.4 & 680.4 \\
\hline Unigene0024355 & Organ-specific protein S2-like & / & 2221.2 & 1084.2 & 307.9 & 264.8 \\
\hline Unigene0072131 & ROTUNDIFOLIA like 7 & AT3G55515 & 56.8 & 26.1 & 12.3 & 10.9 \\
\hline Unigene0053253 & DUF793 domain-containing protein & AT1G01550 & 317.4 & 63.1 & 21.8 & 25.3 \\
\hline Unigene0020203 & F-box protein & AT2G27310 & 283.0 & 99.5 & 43.6 & 42.7 \\
\hline Unigene0027395 & Small ubiquitin-related modifier 2-A-like & AT5G55170 & 967.4 & 239.5 & 96.2 & 199.8 \\
\hline Unigene0035890 & Lectin protein kinase family protein & AT5G24080 & 83.8 & 40.0 & 14.9 & 11.1 \\
\hline \multicolumn{7}{|c|}{ Stress/defense response } \\
\hline Unigene0035676 & Metallothionein-like protein 1 & AT3G09390 & 991.4 & 287.2 & 97.9 & 192.2 \\
\hline Unigene0029269 & Cysteine protease XCP2 & AT1G20850 & 72.0 & 35.3 & 12.6 & 8.9 \\
\hline Unigene0027617 & Pathogenesis-related protein 2 & / & 273.6 & 46.2 & 12.8 & 8.8 \\
\hline Unigene0020874 & Metallothiol transferase FosB & / & 62.9 & 26.3 & 13.0 & 13.5 \\
\hline Unigene0038257 & Isocitrate dehydrogenase & AT1G65930 & 127.3 & 63.6 & 27.5 & 14.6 \\
\hline \multicolumn{7}{|l|}{ Lipid metabolism } \\
\hline Unigene0039640 & Delta(12)-fatty-acid desaturase FAD2-like & AT3G12120 & 100.5 & 44.8 & 20.2 & 7.6 \\
\hline *Unigene0010076 & Delta(12)-fatty-acid desaturase FAD2-like & AT3G12120 & 188.0 & 68.9 & 28.5 & 12.5 \\
\hline *Unigene0053831 & Delta(12) fatty acid desaturase FAD2-like & AT3G12120 & 686.0 & 327.6 & 113.8 & 53.0 \\
\hline *Unigene0014051 & Delta(12)-fatty-acid desaturase FAD2-like & AT3G12120 & 438.8 & 215.2 & 77.5 & 25.0 \\
\hline Unigene0030153 & Delta(12)-fatty-acid desaturase FAD2-like & AT3G12120 & 325.8 & 118.2 & 49.0 & 26.8 \\
\hline Unigene0070830 & PI-PLC X-box domain-containing protein DDB_G0293730 & AT4G38690 & 68.3 & 32.5 & 15.9 & 10.4 \\
\hline Unigene0036376 & DUF563 domain-containing protein & AT2G41640 & 97.6 & 45.9 & 14.2 & 13.8 \\
\hline *Unigene0041208 & CDP-diacylglycerol-glycerol-3-phosphate3-phosphatidyltransferase & AT3G48180 & 182.8 & 82.5 & 31.1 & 15.0 \\
\hline Unigene0022367 & EXORDIUM-like 5 & AT2G17230 & 156.7 & 70.4 & 14.6 & 28.3 \\
\hline Unigene0008870 & GDSL-like Lipase/Acylhydrolase superfamily protein & AT3G26430 & 871.2 & 395.8 & 98.1 & 122.2 \\
\hline \multicolumn{7}{|l|}{ Secondary metabolism } \\
\hline Unigene0034152 & GBR5 & / & 2635.8 & 1186.3 & 411.3 & 465.4 \\
\hline Unigene0033007 & Peroxidase 5-like & AT1G05260 & 219.3 & 40.2 & 15.8 & 29.5 \\
\hline Unigene0027454 & Cytochrome P450 CYP72A219-like & AT3G14630 & 91.4 & 23.7 & 11.1 & 14.3 \\
\hline Unigene0045855 & Caffeoyl-CoA O-methyltransferase & AT4G34050 & 219.5 & 76.9 & 37.6 & 27.5 \\
\hline Unigene0026392 & Polyphenol oxidase & / & 427.5 & 135.0 & 14.7 & 7.5 \\
\hline Unigene0040370 & Shikimate O-hydroxycinnamoyltransferase-like & AT5G48930 & 87.1 & 38.5 & 12.1 & 8.4 \\
\hline Unigene0032140 & Protein WALLS ARE THIN 1-like & AT1G75500 & 205.6 & 40.0 & 19.1 & 26.1 \\
\hline \multicolumn{7}{|l|}{ Cell wall metabolism } \\
\hline \multicolumn{2}{|c|}{ Unigene0010296 Extensin-2-like } & / & 183.0 & 88.0 & 15.5 & 9.7 \\
\hline \multicolumn{2}{|c|}{ Unigene0038129 Extensin-3-like } & AT3G09925 & 414.7 & 162.1 & 12.0 & 9.5 \\
\hline
\end{tabular}

Transporter 
Table 2 Identification of down-regulated DEGs at initial (May) and rapid (July) thickening stages of $P$. notoginseng taproots (Continued)

\begin{tabular}{|c|c|c|c|c|c|c|}
\hline \multirow[t]{2}{*}{ ID } & \multirow[t]{2}{*}{ Description } & \multirow[t]{2}{*}{ TAIR ID } & \multicolumn{4}{|l|}{ RPKM } \\
\hline & & & Mar & May & Jul & Nov \\
\hline Unigene0054315 & Copper-transporting ATPase RAN1-like (HMA7) & AT5G44790 & 58.1 & 25.9 & 12.2 & 26.3 \\
\hline Unigene0031786 & High-affinity nitrate transporter 3.1-like & AT5G50200 & 290.4 & 58.0 & 21.4 & 29.0 \\
\hline Unigene0026653 & Aquaporin TIP2-2 & AT4G17340 & 578.2 & 166.1 & 26.4 & 56.6 \\
\hline Unigene0028381 & Nodulin MtN21-like transporter family protein At5g07050-like & AT5G07050 & 55.2 & 26.5 & 11.2 & 12.1 \\
\hline \multicolumn{7}{|l|}{ Undefined } \\
\hline Unigene0020809 & Uncharacterized protein LOC108212493 & AT2G47485 & 307.8 & 25.5 & 11.5 & 9.9 \\
\hline Unigene0025188 & BnaC05g08810D [Brassica napus] & AT1G13360 & 128.0 & 44.2 & 13.0 & 23.4 \\
\hline Unigene0014035 & Formin-like protein 18 & AT1G10020 & 205.9 & 46.5 & 18.0 & 22.9 \\
\hline Unigene0047041 & Plant organelle RNA recognition domain-containing protein & AT1G57680 & 136.9 & 67.3 & 32.8 & 95.6 \\
\hline Unigene0063477 & Serine/arginine repetitive matrix protein 1 & AT4G02040 & 59.9 & 25.4 & 11.6 & 14.1 \\
\hline Unigene0029630 & Pyridoxal phosphate-dependent transferase & AT5G51920 & 74.9 & 29.1 & 13.1 & 10.7 \\
\hline
\end{tabular}

* Asterisk indicates down-regulated DEGs among initial (May), rapid (July) and stable (November) thickening stages of $P$. notoginseng taproots

regulated in March and November (Fig. 6). To verify whether the gene expression was correlated with hormone metabolism, the endogenous IAA and JA contents at four taproot developmental stages were measured using liquid chromatography-mass spectrometry (LC-MS). The results showed that IAA and JA accumulated predominantly in
March and May and both decreased significantly with the gradual thickening of the taproot, which is consistent with the transcriptome data (Additional file 5: Figure S3; Fig. 6). These results indicated that different hormones may have synergistic or/and antagonistic functions in the regulation of $P$. notoginseng taproot thickening.

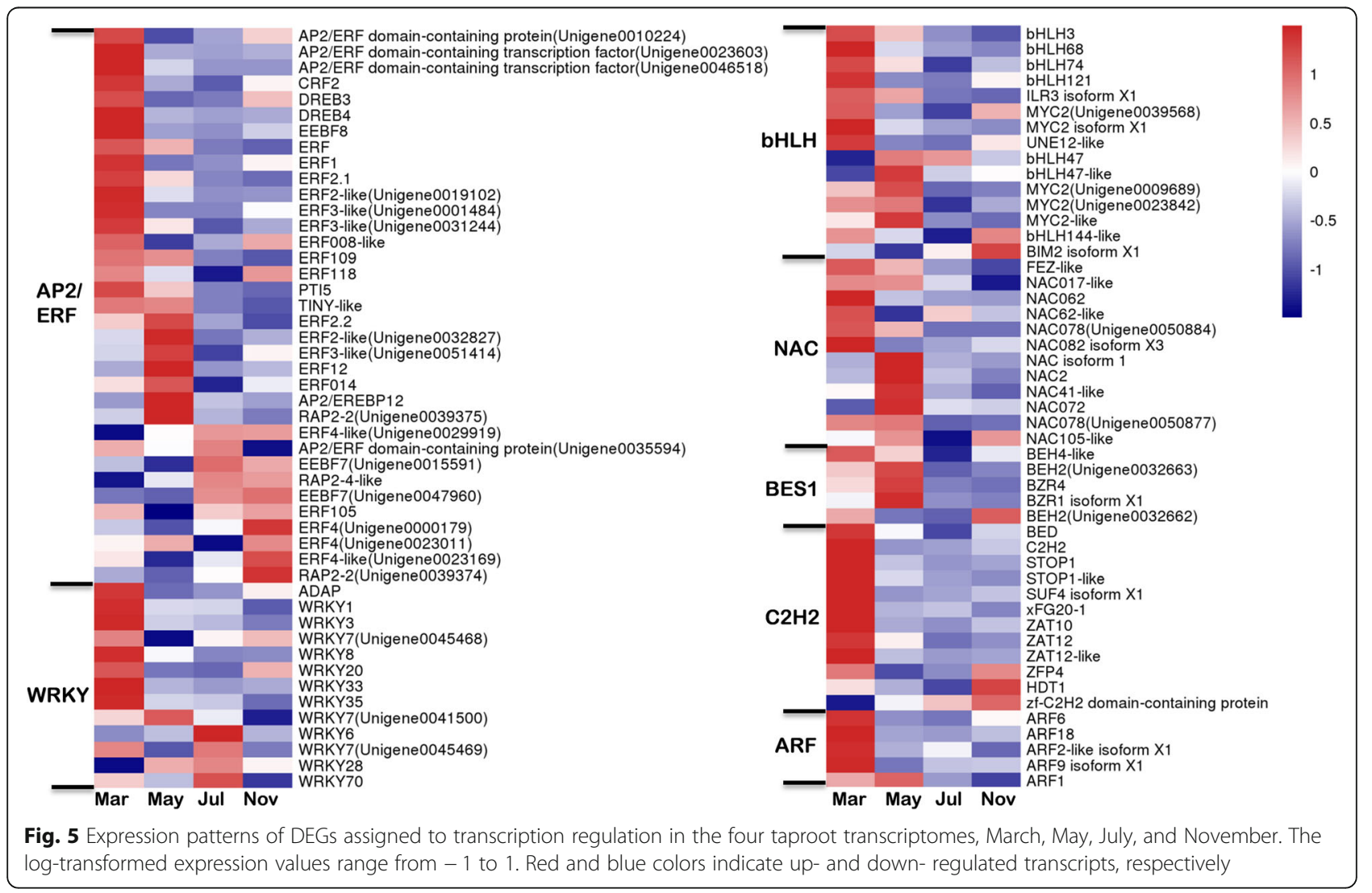




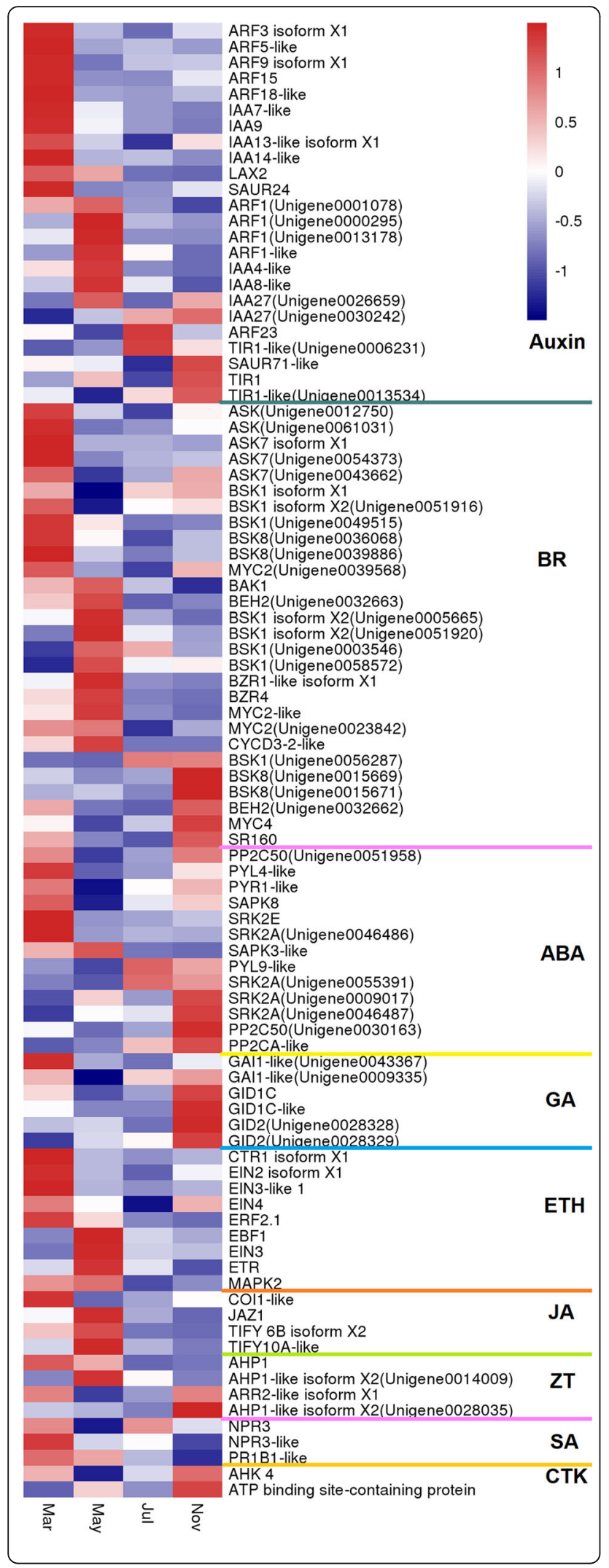

Fig. 6 Expression patterns of DEGs assigned to hormone signal transduction in the four taproot transcriptomes, March, May, July, and November. The log-transformed expression values range from -1 to 1. Red and blue colors indicate up- and down- regulated transcripts, respectively

\section{Differential expression of genes related to starch and sucrose metabolism}

The enrichment of DEGs related to "starch and sucrose metabolism" suggests their important role in $P$. notoginseng taproot thickening (Fig. 4). Metabolic pathway analysis showed that glucose, fructose, sucrose, and starch could be metabolically connected (Fig. 7a). Here, 61 DEGs encoding enzymes related to starch and sucrose metabolism were identified as up-regulated mostly in May, July, and November, including alpha-amylase genes $(A M Y), B A M Y$, starch synthase genes (SS), and granulebound starch synthase genes (GBSS) involved in starch metabolism, and invertase genes (INV), sucrose synthase genes $(\mathrm{SuS})$, and sucrose-phosphate synthase genes (SPS) involved in sucrose metabolism (Fig. 7b). To further clarify whether taproot thickening in $P$. notoginseng is directly associated with changes in carbohydrates, the primary metabolites at four taproot developmental stages were assessed by absolute quantitative analysis using Nuclear Magnetic Resonance (NMR) spectroscopy. PCA revealed that the four samples could be distinctly separated (Additional file 6: Figure S4). Samples collected from July and November clustered closely, suggesting that the overall metabolite profiles were similar between the rapid and stable thickening stages. The differential abundance profiles indicated that five metabolites, including fructose, glucose, sucrose, arginine and malate, differed significantly during taproot thickening (Fig. 8a). Among them, fructose and glucose accumulated dominantly in March and May, while sucrose accumulated mostly in May, July, and November. This was basically consistent with the transcriptome data (Fig.7b; Fig. 8a). It is presumed that sucrose is degraded into fructose and glucose for conversion into other organic matters during the early stage, while taproot thickening is also accompanied by increased malate and arginine accumulation (Fig. 8b).

\section{Differential expression of cell wall metabolism transcripts}

The cell wall not only strengthens the plant body, but also has key roles in plant growth, cell differentiation, cell expansion, intercellular communication, water movement and defense [31,32]. In the present study, a total of 96 DEGs encoding enzymes that are involved in cell wall modification, synthesis, and degradation were identified. Among them, the largest number of genes was up-regulated in May (Fig. 9), suggesting that the changes in cell wall components are necessary for the initiation and induction of taproot thickening in the early stages in P. notoginseng. In particular, 

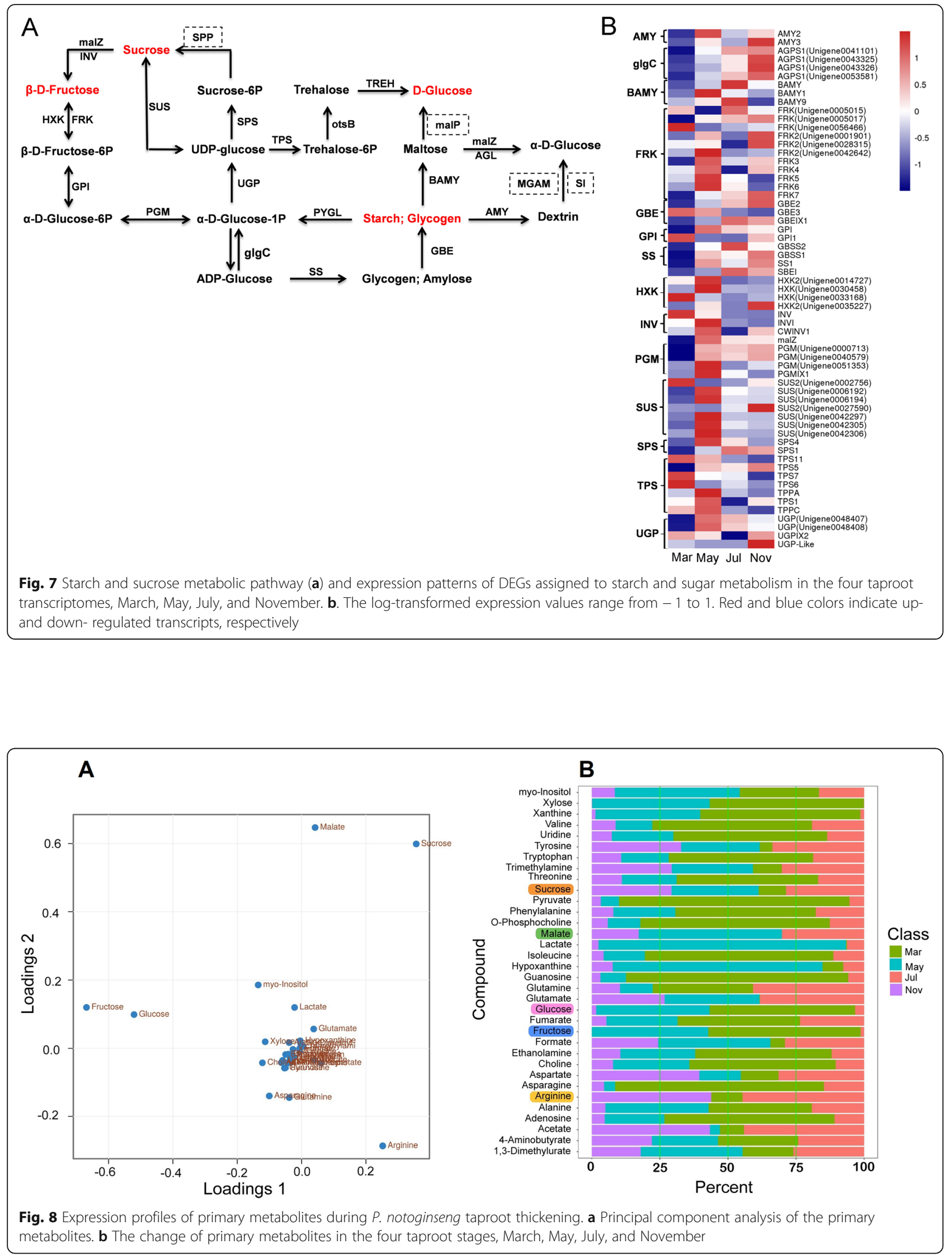

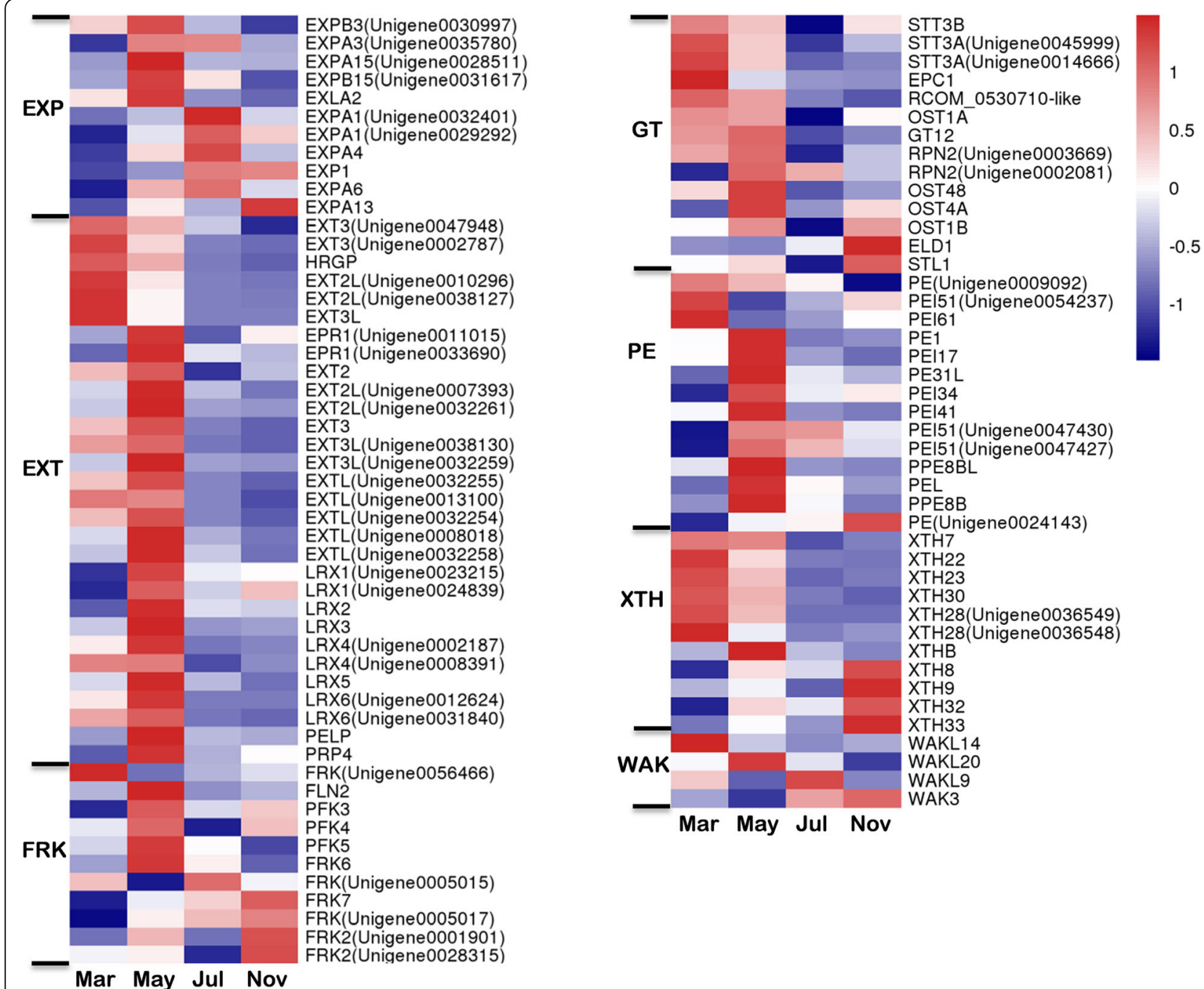

Fig. 9 Expression patterns of DEGs assigned to cell wall metabolism in the four taproot transcriptomes, March, May, July, and November. The logtransformed expression values range from -1 to 1 . Red and blue colors indicate up- and down- regulated transcripts, respectively

DEGs encoding extension (EXT), glycosyltransferases (GT), and pectinesterase (PE) were mostly up-regulated in March and May, while those encoding expansin (EXP) were highly expressed in May and July (Fig. 9).

\section{Discussion}

Elucidating the molecular mechanisms associated with taproot thickening is important for improving the yield and quality of $P$. notoginseng products. Here, taproot development in one-year-old $P$. notoginseng plants at four critical stages was analyzed using an integrated morphological, transcriptomics, and metabonomics approach. We provided the framework for the gene expression changes involved in $P$. notoginseng taproot thickening, thus providing a foundation for the future elucidation of the molecular mechanisms underlying taproot growth and development in P. notoginseng.

\section{Potential DEGs playing critical roles in $P$. notoginseng} taproot thickening

We found that taproot thickening in $P$. notoginseng was associated with vascular cambium activity, resulting in the production of the secondary xylem and phloem (Fig. 2). The further growth of the secondary xylem and secondary phloem is dependent on cell division, expansion, and differentiation and results in a rapid increase in root diameter; a process controlled by plant hormones, TFs, and metabolic pathways [33, 34].

\section{Hormonal signaling plays multidirectional roles in $P$. notoginseng taproot thickening}

The plant hormone-mediated signaling pathway was the most significantly enriched pathway according to the KEGG pathway analysis (Fig. 4), as reflected by as many as 93 DEGs that were related to plant hormone signaling 
pathways (Fig. 6). Our study showed that the endogenous IAA content and genes involved in IAA signaling, including auxin/indole-3-acetic acid (Aux/IAAs) and auxin response factors (ARFs), were significantly up-regulated in the early stage and down-regulated in the later stage during P. notoginseng taproot thickening (Fig. 6; Additional file 5: Figure S3). These results suggested that IAA plays potential roles in the initial thickening of the $P$. notoginseng taproot, and degrading IAA and maintaining it at a lower concentration is necessary for promoting the continued thickening of the taproot. Congruent with our findings, previous studies in sweet potato (Ipomoea batatas) showed that auxin levels have been found to be elevated during early tuberous root formation $[1,35,36]$. The later stage of tuberous root development was positively correlated with concentrations of $\mathrm{ABA}$ and $\mathrm{CK}$ levels, but not with IAA levels [35].

It is unsurprising that the expression of genes related to the GA signaling pathway was significantly enhanced in November (Fig. 6). In general, GA is a negative regulatory hormone in storage organ formation and inhibits tuberization in potato [37]. Overexpression of the GA oxidase (GAox) gene could increase GA content, which promoted stolon elongation and inhibited storage organ formation [38]. Further research showed that StPP2Ac2b, a serine/threonine protein phosphatases type $2 \mathrm{~A}$ (PP2A) from potato, acts in the stolons as a positive regulator of tuber induction by modulating the expression of StGA2ox1 [39]. Xu et al. (1998) also observed that a high content of exogenous GA inhibited storage organ formation and promoted stolon elongation. Endogenous GA levels were high during stolon elongation and decreased when the stolon tips started to swell under inducing conditions in potato [40]. Our findings suggest that the initiation of thickening in the taproot of $P$. notoginseng is regulated by the crosstalk between IAA and GA.

In addition, the expression of genes related to the $\mathrm{ABA}$ signaling pathway was significantly enhanced in November (Fig. 6). In general, ABA accumulates during autumn. While it is possible that ABA-responsive genes are upregulated in response to winter dormancy, it is known that $\mathrm{ABA}$ antagonizes GA, as demonstrated by the finding that ABA stimulates potato tuberization by counteracting GA [40-42]. In fact, recent studies have discovered the novel antagonizing roles of GA and ABA in integrating growth and development in plants with environmental signaling $[43,44]$. These results suggest that antagonistic interactions between ABA and GA signaling potentially modulate taproot thickening in $P$. notoginseng.

Transcriptional regulation is a basic regulatory factor for $P$. notoginseng taproot thickening

In Arabidopsis, ERF109, ERF114, and ERF115 were reported to positively regulate quiescent center (QC), distal stem cells (DSCs), and cell differentiation by mediating reactive oxygen species (ROS) signaling [45]. Here, a gene homologous to Arabidopsis ERF109 was significantly upregulated in March and May (Fig. 5). Recent studies have further demonstrated that the highly JA-responsive ERF109 mediates the cross-talk between JA signaling and IAA biosynthesis to regulate root development in Arabidopsis $[46,47]$. Moreover, JA induces the expression of ERF109 and ERF115, which regulates the asymmetric cell division of root stem cells and QC quiescence by the RETINOBLASTOMA-RELATED (RBR)-SCARECROW (SCR)-SHORT ROOT (SHR) protein network [47]. Interestingly, we also found that endogenous JA content and genes associated with JA signaling were significantly increased in the early stage of taproot development (Fig. 6; Additional file 5: Figure S3), suggesting that JA-induced ERF transcriptional regulation initiates the transition from seed roots to taproot thickening in $P$. notoginseng.

Furthermore, some genes encoding WRKY (e.g., WRKY3, 8), NAC (e.g., NAC1,2), MYC (e.g., MYC2), and ARF (e.g., ARF9) were up-regulated at the early stage of taproot development (Fig. 6). It was reported that MYC and NAC TFs might control secondary cell wall metabolism [48, 49]. Additionally, some NAC TFs have also been shown to be involved in the development of the secondary xylem $[50,51]$ and the regulation of the transition from cell division to cell expansion [52]. All these studies suggest that these TFs may be potentially involved in taproot thickening in $P$. notoginseng, but their exact roles require further exploration. In addition, we also found that a gene encoding DA1-related proteins was significantly up-regulated during the taproot thickening process (Table 1). DA1-related genes encode proteins containing a LIM domain and zinc finger-acting elements. In Arabidopsis, DAR2 functions in the regulation of root stem cell niche activity and root meristem size by maintaining the normal auxin distribution mediated by auxin transport, while DAR3 mediates proper root system architecture by unloading carbohydrates from the phloem to the root tip [53, 54], suggesting that the distribution of auxin rather than its content is more important for taproot thickening in P. notoginseng.

\section{Carbohydrate and storage protein metabolism provides materials for $P$. notoginseng taproot thickening}

We found that "starch and sucrose metabolism pathways" was significantly activated during the development of the P. notoginseng taproot (Fig. 4). Sucrose cleavage is vital to multicellular plants for carbon metabolism and plant sink development. The reactions of SuS and INV catalyze sucrose cleavage, among which SuS can reversibly catalyze sucrose to UDP-glucose and fructose, while INV catalyzes sucrose to glucose (Glu) and fructose [55]. Here, genes encoding SuS and INV were mostly up- 
regulated in March and May (Fig. 7b), suggesting that sucrose catabolism is required for the thickening of $P$. notoginseng taproots. This was further confirmed by the discovery that glucose and fructose were abundantly accumulated, while sucrose was low, in March and May (Fig. 8b). Similar results were obtained in a previous study, and SuSy is a key enzyme involved in the early development of the storage root of radish [56].

Starch is considered to be one of the major storage carbohydrates. The accumulation of starch proceeds simultaneously with the swelling of the storage organs in potato, lotus (Nelumbo nucifera), and cassava [12, 37, 57]. Here, genes encoding SBE, GBSS, SS, and alpha-1,4glucan phosphorylase L isozymes were significantly upregulated in July and November (Fig. 7b; Table 1), implying that starch accumulation in an active sink plays a major role in the rapid and stable thickening stages. However, we also found that glucose and fructose were consumed in excess, while sucrose increased slightly, with taproot thickening (Fig. 8b), leading us to speculate that metabolic transition from starch to sucrose is required during the later stages of taproot thickening. This is based on the fact that genes encoding SPS were up-regulated in the rapid and stable thickening stages (Fig. 7b). This result is similar to that in previous studies on radish where up-regulated SPS was found to play a major role in the taproot thickening stage [8]. In fact, Jackson [58] noticed that a high content of sucrose is also a necessary condition during storage organ formation. In potato, sugars are thought to act as drivers of the formation and growth of the sink tuber [59]. These results indicate that the homeostasis and feedback regulation of starch and sucrose are required for the process of $P$. notoginseng taproot thickening, playing different roles at different thickening stages through dynamic metabolism.

Several lines of evidence suggest that storage proteins might be associated with the formation of underground storage organs [60-62]. Storage proteins that are localized in the vacuole and expressed mainly in the tuber or core have been reported in some species, such as patatin in potato and sporamin in sweetpotato [60]. In this study, genes encoding RNase-like major storage protein and vacuolar protein sorting-associated protein 32 homolog 2 were found to be significantly up-regulated during the process of $P$. notoginseng taproot thickening. (Table 1). A previous study showed that ginseng major protein (GMP) is a specific RNase-like storage protein in Panax ginseng roots and is thought to be highly correlated with the seasonally-regulated taproot development of $P$. ginseng [63]. Functional analysis of this candidate gene might be useful for the genetic engineering or marker-assisted selection of new $P$. notoginseng cultivars with enlarged taproots.
We also discovered that malate and arginine changed significantly during the $P$. notoginseng taproot thickening process (Fig. 8b). Malate, an important intermediate product of carbon metabolism in the citric acid cycle (TCA) and glyoxylic acid cycle, not only synthesizes some metabolites but also provides energy for plant metabolism [64]. Furthermore, it was also reported that malate can function as an intercellular diffusion factor, affecting the cell wall expansion of the roots [65]. Comparatively, arginine was mostly accumulated in the rapid and stable thickening stages (Fig. 8b). A recent study has shown that arginine produced by argininosuccinate lyase (ASL) is required for normal root elongation in rice, which is supported by the fact that the exogenous addition of arginine but not other amino acids rescued root growth in the asl mutant [66]. As the taproot of $P$. notoginseng was also significantly elongated during these two stages (Fig. 1b), we suspect that arginine might be involved in the taproot cell elongation of $P$. notoginseng. However, the specific acting mechanism is unclear, and there is only minor evidence that arginine is an essential substrate for the synthesis of protein and nitric oxide (NO, cell-signaling molecules) and the transport and storage of nitrogen in plants [67].

\section{The regulatory networks associated with $P$. notoginseng taproot thickening}

Taproot thickening in $P$. notoginseng is a complex regulatory process that is affected by many factors, including signal transduction pathways (hormone and transcription signaling) and metabolism processes (carbohydrate, storage, energy metabolism and cell wall). In this study, a putative model of the regulatory network associated with $P$. notoginseng taproot thickening was proposed by integrating both transcriptomics and metabonomics analysis with previous research findings. As illustrated in Fig. 10, hormones play a multidirectional role in the process of taproot thickening in $P$. notoginseng, involving the synergistic and antagonistic regulation of many hormones at different developmental stages. IAA mainly plays a role in the initial thickening stage. Persistent taproot thickening may be closely related to lower auxin levels and proper distribution, while GA may play a role in the later stage of taproot thickening by antagonizing ABA signaling. TFs mainly mediate transcriptional regulation in the initial thickening stage, and are closely associated with hormone signaling, such as early JA signaling mediation by ARFs. In the initial thickening stage, sucrose is hydrolyzed into fructose and glucose to synthesize starch for the rapid and stable thickening of the taproot, while the remaining glucose and fructose are re-synthesized into sucrose, which coordinates with starch to drive taproot thickening. Due to the prevailing sucrose-starch metabolism, lipids and other secondary 


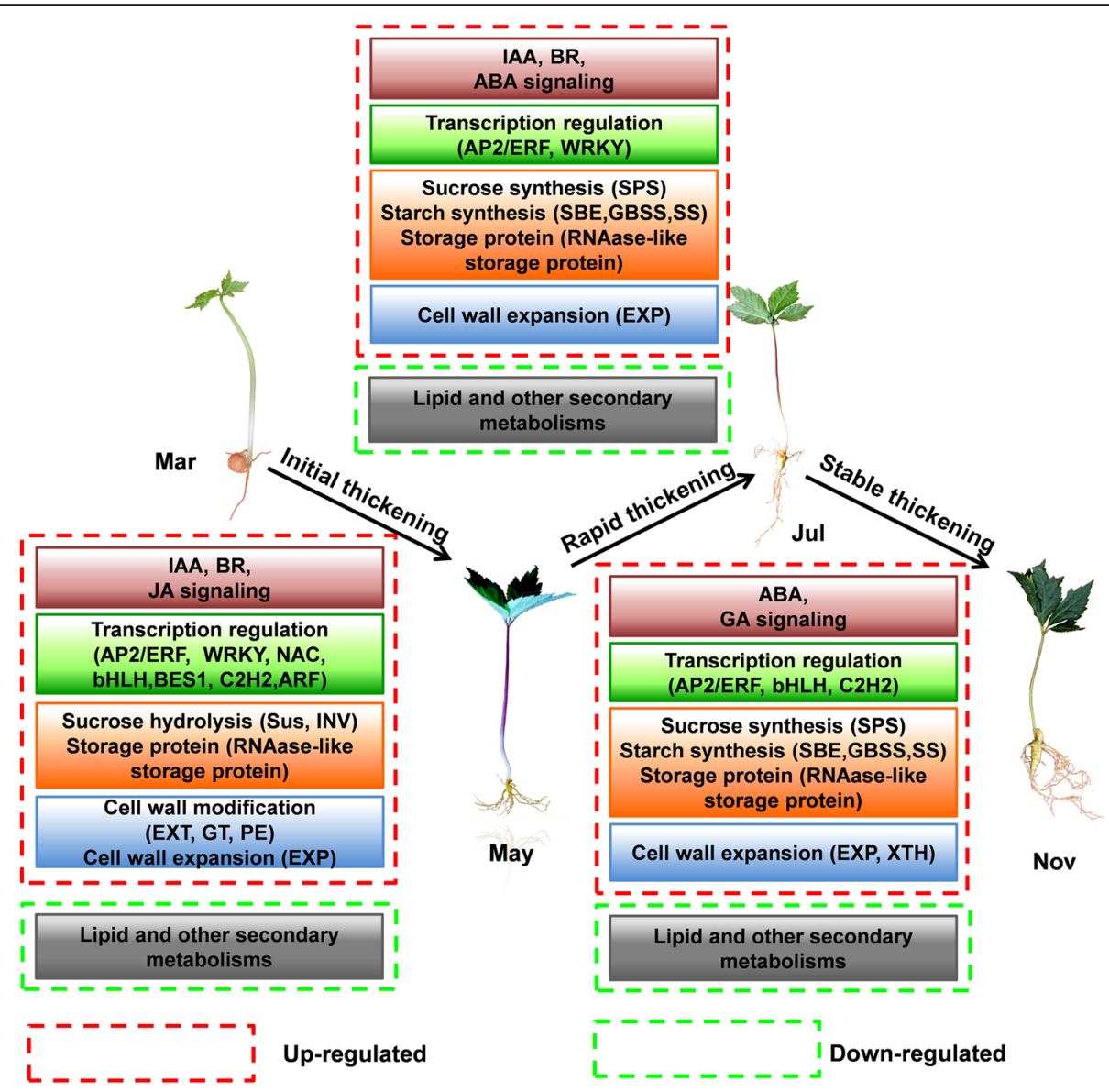

Fig. 10 A proposed model of genetic and molecular interactions in the regulatory network during $P$. notoginseng taproot thickening

metabolites (e.g., lignin) are down-regulated, thus coordinating the direct carbon flux into starch. In addition, storage proteins (e.g., RNase-like major storage protein), malate and arginine also play an important role in unknown functions during the taproot thickening process. Additionally, the modification and relaxation of the cell wall structure in the initial thickening stage contribute further to the continued thickening by EXP-mediated cell wall expansin. Taken together, these results implied that all these differentially expressed genes (DEGs) could play important roles in the regulatory network of $P$. notoginseng taproot thickening. Following further functional validation, these critical genes could greatly contribute to the manipulation of the shape, yield, and quality of the $P$. notoginseng taproot.

\section{Conclusions}

Comparative transcriptome and metabolome changes at four critical developmental stages in the taproots of $P$. notoginseng were systematically investigated in this study. A total of 29,957 transcripts were obtained, and the DEGs during the taproot thickening process were identified for the first time. GO and KEGG pathway enrichment analysis revealed that these DEGs were mainly involved in processes of "plant hormone signal transduction," "starch and sucrose metabolism," and "phenylpropanoid biosynthesis." Furthermore, integrated analysis of the DEGs, endogenous hormones, and primary metabolites provided a hypothetical model of the genetic regulatory network, highlighting that certain specific signal transduction pathways and metabolic processes are associated with taproot thickening in $P$. notoginseng. These findings not only potentially accelerate the process of genetic improvement of new $P$. notoginseng cultivars with enlarged taproots but also provide novel insights into the molecular regulatory mechanisms underlying root morphogenesis in this Chinese medicinal herb with enlarged roots.

\section{Methods}

\section{Plant materials}

One-year-old $P$. notoginseng plants at eight developmental stages, including March, May, June, July, August, September, October, and November were collected from the Xiaoxinzhai planting base (east longitude 104 $24^{\prime}$ $29.0484^{\prime \prime}$, north latitude $\left.23^{\circ} 24^{\prime} 8.496^{\prime \prime}\right)$. They were planted 
by Wenshan Miao Xiang P. notoginseng Industrial Co., Ltd. For hormone detection, primary metabolite assays, and RNA-Seq, the collected seedlings were gently washed with clean water, and the shoots and fibrous roots were rapidly excised. The taproots were then placed in liquid nitrogen immediately and stored in an ultra-cold storage freezer at $-80^{\circ} \mathrm{C}$ until further use.

\section{Determination of growth indexes and total saponin}

The diameter of the taproot was measured using a Vernier caliper. The fresh weight of the taproots was weighed using an electronic balance, following which the dry weight was determined after drying for 3-5 days at $60^{\circ} \mathrm{C}$ in an oven. For anatomical structure observation, taproots were cut into 2 -mm-thick slices and immediately fixed in $5 \mathrm{~mL}$ formalin-glacial acetic acid-alcohol (FAA) solution for $48 \mathrm{~h}$. Paraffin embedding was then performed as described by Canene-Adams [68]. For total saponin detection, dry taproots were ground into powder (through 80 mesh sieve), following which $0.6 \mathrm{~g}$ powder was added to $50 \mathrm{~mL} 70 \%$ methanol, left to stand for about $30 \mathrm{~min}$, and then ultrasonicated for $30 \mathrm{~min}$. The filtered samples were analyzed by high-performance liquid chromatography (HPLC). The chromatographic conditions were as follows: column $(250 \times 4.6,5 \mathrm{~m})$, acetonitrile (A) - water (B) as the mobile phase, detection wavelength $203 \mathrm{~nm}$, and flow rate $1.5 \mathrm{~mL} / \mathrm{min}$.

\section{RNA library construction and sequencing}

The taproots of one-year-old $P$. notoginseng plants collected in March, May, July, and November were used for RNA-Seq. Each sampling point had three biological replicates. After the total RNA was extracted, the eukaryotic mRNA was enriched by Oligo (dT) beads, while prokaryotic mRNA was enriched by removing rRNA by a RiboZeroTM Magnetic Kit (Epicentre). The enriched mRNA was fragmented into short fragments using fragmentation buffer and then reverse transcribed into cDNA with random primers. Second-strand cDNA was synthesized by DNA polymerase I, RNase $\mathrm{H}$, dNTPs, and buffer. The cDNA fragments were then purified using a QiaQuick PCR extraction kit, and following end-repair and the addition of poly $(\mathrm{A})$ the fragments were ligated to the Illumina sequencing adapters. The ligation products were selected by agarose gel electrophoresis, PCRamplified, and then sequenced using the Illumina HiSeqTM 4000 at Gene Denovo Biotechnology Co. (Guangzhou, China).

\section{Analysis of the RNA-Seq data}

Clean data were obtained by removing reads containing adapters, poly- $\mathrm{N}$, and low-quality reads from the raw data. The high-quality clean reads were mapped to ribosome RNA (rRNA) to identify residual rRNA reads. The high-quality clean reads were then mapped to the reference transcriptome using the short reads alignment tool Bowtie 2 with the default parameters [69], and the mapping ratio was calculated. The gene abundances were calculated and normalized to RPKM (Reads Per kb per Million reads) [70]. To identify DEGs across samples or groups, the edgeR package (http://www.r-project.org/) was used. Reads with $\log 2 \mid$ Fold Change $\mid>1$, FDR $<0.05$ and RPKM values $>10$ were selected to annotate DETs. DEGs were then subjected to enrichment analysis of GO functions and KEGG pathways.

\section{Determination of endogenous IAA and JA contents}

The taproots of one-year-old P. notoginseng plants collected in March, May, July and November were used to determine the IAA and JA contents. Standard calibration curves were constructed for IAA and JA to obtain two standard curve samples with concentrations ranging from $0.5 \mathrm{ng} / \mathrm{mL}$ to $1000 \mathrm{ng} / \mathrm{mL}$. A mixed-standard solution containing $5 \mathrm{ng} / \mathrm{mL}$ of each of the deuteriumlabeled plant hormones was prepared as the internal standard (IS) solution. A quality control (QC) sample was prepared by mixing plant hormone standards with the IS to obtain a final concentration of $10 \mathrm{ng} / \mathrm{mL}$ and 5 $\mathrm{ng} / \mathrm{mL}$. The QC sample was used to monitor the precision and stability of the instrument during the experiment runtime. One-hundred milligrams of sample was added to liquid nitrogen and $10 \mu \mathrm{L}$ of IS was added to the sample in $1 \mathrm{~mL}$ extracting solution. The samples were vortexed for $30 \mathrm{~s}$ and placed in a shaker for $30 \mathrm{~min}$ in the dark. Two milliliters of dichloromethane was added to the mixture, which was then shaken in the dark for $30 \mathrm{~min}$. Samples were centrifuged for $5 \mathrm{~min}$ at $4{ }^{\circ} \mathrm{C}$ at $3500 \mathrm{rpm}$. After carefully collecting the lower layer into a clean centrifuge tube, another $0.5 \mathrm{~mL}$ of dichloromethane was added to the sample and vortexed for $30 \mathrm{~s}$. Samples were centrifuged for $5 \mathrm{~min}$ with the same settings as described above, and the lower layer was collected again. Both portions of the lower layer solution were combined, and solvent was evaporated under nitrogen. The purified samples were redissolved in $0.5 \mathrm{~mL}$ of $0.05 \%$ methanol and vortexed for $30 \mathrm{~s}$. Solid particles were removed by first centrifuging at $3500 \mathrm{rpm}$ for 10 min at $4{ }^{\circ} \mathrm{C}$ and then passing through a $0.22 \mu \mathrm{m}$ filter. Filtrates were collected for LC-MS analysis.

\section{Primary metabolite analysis}

The taproots of one-year-old $P$. notoginseng plants collected in March, May, July, and November were used to determine the primary metabolites by NMR. Briefly, freeze-dried plant material was weighed and suspended in a $1000 \mu \mathrm{L}$ of $50 \% / 50 \%$ methanol water solution. A $4 \mathrm{~s}$ on/off cycling program was used (eight cycles) for the in-solution ultrasonic extraction process (Sonics VX- 
130, USA). Samples were centrifuged at $13,000 \mathrm{rpm}$ for $15 \mathrm{~min}$, following which the supernatant was lyophilized and then re-dissolved in $450 \mu \mathrm{L}$ of water. Four-hundred and fifty microliters of the aqueous layer was transferred to a clean $2 \mathrm{~mL}$ centrifuge tube following which $50 \mu \mathrm{L}$ DSS standard solution (Anachro, Canada) was added. Samples were mixed well before being transferred to a 5 mm NMR tube (Norell, USA). Spectra were collected using a Bruker AV III $600 \mathrm{MHz}$ spectrometer. The first increment of a $2 \mathrm{D}-1 \mathrm{H}, 1 \mathrm{H}-\mathrm{NOESY}$ pulse sequence was utilized for the acquisition of ${ }^{1} \mathrm{H}-\mathrm{NMR}$ data and for suppressing the solvent signal. Experiments used a $100 \mathrm{~ms}$ mixing time along with a $990 \mathrm{~ms}$ pre-saturation $(\sim 80 \mathrm{~Hz}$ gammaB1). Spectra were collected at $25^{\circ} \mathrm{C}$, with a total of 128 scans over a period of $15 \mathrm{~min}$.

\section{RT-qPCR analysis}

Quantitative real-time PCR (RT-qPCR) was employed to validate the quality of RNA-seq results. Total RNAs from taproot samples at four stages including March, May, July, and November were extracted following the manufacturer's instructions (Magen Biotech Co., Ltd., China) and reverse transcribed to cDNA following the manufacturer's instructions (Vazyme Biotech Co.,Ltd., China). Each reaction was carried out using $10 \mu \mathrm{L} 2 \times$ SYBR green reaction mix (Vazyme Biotech Co.,Ltd., China), $1.0 \mu \mathrm{L}$ diluted $\mathrm{cDNA}$, and $0.4 \mu \mathrm{L}$ of each primer in a total volume of $20 \mu \mathrm{L}$ system. RT-qPCR amplification reactions were performed in an ABI QuantStudio 5 Real-Time PCR System (Applied Biosystems). The specific primers used for RT-qPCR were designed with Primer3 web version 4.1.0 software, which are listed in Additional file 7: Table S3. Three technological replicates for each gene were performed and P.notoginseng YLS8 was used as an endogenous control. The relative gene expression value was calculated with the $2^{-\Delta \Delta C T}$ method.

\section{Supplementary information}

Supplementary information accompanies this paper at https://doi.org/10. 1186/s12870-019-2067-5.

Additional file 1: Table S1. The pairwise compared DEGs of four developmental stages in $P$. notoginseng taproots.

Additional file 2: Figure S1. Functional categories of up- and downregulated unigenes at each developmental stage of $P$. notoginseng taproots. The classification was preformed according to gene ontology (GO) biological process.

Additional file 3: Figure S2. qRT-PCR verification diagram of DEGs during the thickening process in the taproots of $P$. notoginseng. $\mathbf{a}$ The expression levels determined by qRT-PCR and RNA-seg from four stages. $\mathbf{b}$ Correlation of gene expression ratio between RNA-Seq results (RPKM) and $\mathrm{GPCR}\left(2^{-\Delta \Delta \mathrm{Ct})}\right.$ results. Results were calculated using $\log _{2}$ (Fold Change) values. The $r$ value indicates the correlation coefficient. ${ }^{* *}$ indicates significant difference at $p \leq 0.01$.
Additional file 4: Table S2. Statistics analysis of TFs gene expression in taproot thickening in $P$. notoginseng.

Additional file 5: Figure S3. Change of endogenous indole-3-acetic acid (IAA) and jasmonate (JA) contents in taproot thickening in P. notoginseng. Results are shown as mean expression \pm standard deviation of three independent experiments.

Additional file 6: Figure S4. Principal component analysis of primary metabolites in taproot thickening in P. notoginseng. Each sample contains five biological repeats.

Additional file 7: Table S3. Primers for qRT-PCR validation of candidate genes.

\section{Abbreviations}

ABA: Abscisic acid; ABC: ATP-bindingcassette transporter; AMY: Alphaamylase genes; AP2/ERF: APETALA2/ethylene-responsive factor; APRR7: Arabidopsis PSEUDO-RESPONSE REGULATOR 7; ARF: Auxin response factor; ASL: Argininosuccinate lyase; Aux/IAA: Auxin/Indole-3-acetic acid; BAMY: Beta-amylase; BES: BRI1-EMS-suppressor; bHLH: Basic helixloop-helix transcription factor; BR: Brassinosteroid; bZIP: Basic leucine zipper; C2H2: Cys2-His2 zinc finger protein; CDC5: Cell division cycle 5-like protein; CLE: CLAVATA3/ESR-related gene; CTK: Cytokinin; DAR: DA1-related protein; DEG: Differentially expressed gene; DSC: Distal stem cells; ETH: Ethylene; EXP: Expansin; EXT: Extension; GA: Gibberellin; GAox: GA oxidase; GBE: 1,4alpha-glucan-branching enzyme-like isoform X1; GBSS: Granule-bound starch synthase genes; GGR: Heterodimeric geranylgeranyl pyrophosphate synthase small subunit; GMP: Ginseng major protein; GO: Gene ontology; GT: Glycosyltransferases; HDS: 1-hydroxy-2-methyl-2-(E)-butenyl 4diphosphate synthase; HMGR: 3-hydroxy-3-methylglutaryl-CoAreductase; IAA: Indole-3-acetic acid; INV: Invertase genes; JA: Jasmonate; JAZ1: Jasmonate ZIM-domain 1; KEGG: Kyoto encyclopedia of genesand genomes; LC-MS: Liquid chromatography-mass spectrometry; MVDD: Mevalonate diphosphate decarboxylase; MVK: Mevalonate kinase; NAC: NAM, ATAF, and CUC (NAC) transcription factors; NF-YA2: Nuclear transcription factor $Y$ subunit A-2 protein; NMR: Nuclear Magnetic Resonance; NO: Nitric oxide; PE: Pectinesterase; PHO1: Alpha-1,4 glucan phosphorylase L isozyme; PMP: RNase-like major storage protein; PP2A: Serine/threonine protein phosphatases type 2A; QC: Quiescent center; RBR: Retinoblastomarelated; ROS: Reactive oxygen species; SA: Salicylic acid; SBE: Starch branching enzyme; SHR: Short root protein; SPS: Sucrose-phosphate synthase genes; SuS: Sucrose synthase genes; TCA: Tricarboxylic acid cycle;

TF: Transcription factor; VPS32: Vacuolar protein sorting-associated protein 32 homolog 2; XTH: Xyloglucan endotransglucosylase/hydrolase protein; ZT: Zeatin

\section{Acknowledgments}

We thank LetPub (www.letpub.com) for its linguistic assistance during the preparation of this manuscript.

\section{Authors' contributions}

WF, SCY, GHZ and BH conceived the study. XJL, JLY, YCL, ZLQ, YL, SY, JRT, $M C, G Q L, Y Z$ and JWC performed the experiments and carried out the analysis. XJL, WF, SCY, and JLY designed the experiments and wrote the manuscript. All the authors approved the manuscript.

\section{Funding}

This work was supported by the National Key Reseach and Development Plant (No.2017YFC1702500) for material collection and RNA-seq, the Major Science and Technique Programs in Yunnan Province (No.2016ZF001) for determination of primary metabolite and hormone, the National Natural Science Foundation of China (No. U1402262) for bioinformatic analysis, the Yunnan Ten Thousand Talents Plan Young \& Elite Talents Project (YNWRQNBJ-2018-047) and the project of young and middle-aged talent of Yunnan province (No. 2019HB019) for writing and publishing manuscript.

\section{Availability of data and materials}

All data generated or analyzed during this study are included in this published article and its supplementary information files. 


\section{Ethics approval and consent to participate}

Not applicable. The authors declared that experimental research works on the plants described in this paper comply with institutional, national and international guidelines.

\section{Consent for publication}

Not applicable.

\section{Competing interests}

The authors declare that they have no competing interests.

\section{Author details}

${ }^{1}$ State Key Laboratory of Conservation and Utilization of Bio-resources in Yunnan, The Key Laboratory of Medicinal Plant Biology of Yunnan Province, National\& Local Joint Engineering Research Center on Germplasm Innovation \& Utilization of Chinese Medicinal Materials in Southwest China, Yunnan Agricultural University, Kunming 650201, China. ${ }^{2}$ College of Horticulture and Landscape, Yunnan Agricultural University, Kunming 650201, China. ${ }^{3}$ State Key Laboratory of Plant Physiology and Biochemistry, College of Life Sciences, Zhejiang University, Hangzhou, China.

Received: 4 June 2019 Accepted: 2 October 2019

\section{Published online: 26 October 2019}

\section{References}

1. Noh SA, Lee HS, Huh EJ, Huh GH, Paek KH, Shin JS, et al. SRD1 is involved in the auxin-mediated initial thickening growth of storage root by enhancing proliferation of metaxylem and cambium cells in sweetpotato (Ipomoea batatas). J Exp Bot. 2010;61(5):1337-49.

2. Loretan PA, Bonsi CK, Mortley DG, Wheeler RM, Mackowiak CL, Hill WA, et al. Effects of several environmental factors on sweet potato growth. Adv Space Res. 1994;14(11):277-80.

3. Mortley D, Hill J, Loretan P, Bonsi C, Hill W, Hileman D, et al. Elevated carbon dioxide influences yield and photosynthetic responses of hydroponically-grown sweetpotato. Acta Hortic. 1996;440:31-6.

4. Eguchi T, Kitano M, Eguchi H. Growth of sweetpotato tuber as affected by the ambient humidity. Biotronics. 1998;27:93-6.

5. Kano Y, Ming ZJ. Effects of soil temperature on the thickening growth and the quality of sweetpotato during the latter part of their growth. Environ Control Biol. 2000;38(3):113-20.

6. van Heerden PDR, Laurie R. Effects of prolonged restriction in water supply on photosynthesis, shoot development and storage root yield in sweet potato. Physiol Plantarum. 2008;134(1):99-109.

7. Mitsui Y, Shimomura M, Komatsu K, Namiki N, Shibata-Hatta M, Imai M, et al. The radish genome and comprehensive gene expression profile of tuberous root formation and development. Sci Rep. 2015:5:10835.

8. Yu R, Xu L, Zhang W, Wang Y, Luo X, Wang R, et al. De novo taproot transcriptome sequencing and analysis of major genes involved in sucrose metabolism in radish (Raphanus sativus L.). Front Plant Sci. 2016;7:585.

9. Xie $Y, X u$, Wang $Y$, Fan $L$, Chen $Y$, Tang M, et al. Comparative proteomic analysis provides insight into a complex regulatory network of taproot formation in radish (Raphanus sativus L.). Hortic Res. 2018;5:51.

10. $Y u$ R, Wang $Y, X u L$, Zhu X, Zhang W, Wang R, et al. Transcirptome profiling of root microRNAs reveals novel insights into taproot thickening in radish (Raphanus sativus L.). BMC Plant Biol. 2015;15:30.

11. Zhang C, Zhang H, Zhan Z, Liu B, Chen Z, Liang Y. Transcriptome analysis of sucrose metabolism during bulb swelling and development in onion (Allium cepa L.). front. Plant Sci. 2016;7:1425.

12. Wang $X$, Chang $L$, Tong $Z$, Wang D, Yin Q, Wang D, et al. Proteomics profiling reveals carbohydrate metabolic enzymes and 14-3-3 proteins play important roles for starch accumulation during cassava root tuberization. Sci Rep. 2016;6:19643.

13. Teo CJ, Takahashi K, Shimizu K, Shimamoto K, Taoka Kl. Potato tuber induction is regulated by interactions between components of a tuberigen complex. Plant Cell Physiol. 2017;58(2):365-74.

14. Pal T, Malhotra N, Chanumolu SK, Chauhan RS. Next-generation sequencing (NGS) transcriptomes reveal association of multiple genes and pathways contributing to secondary metabolites accumulation in tuberous roots of Aconitum heterophyllum wall. Planta. 2015;242(1):239-58.
15. Liu $H$, Wang $Y$, Wang $T$, Ying $X$, Wu $R$, Chen $H$. De novo assembly and annotation of the Zhe-Maidong (Ophiopogon japonicus (L.f.) Ker-Gawl) transcriptome in different growth stages. Sci Rep. 2017;7(1):3616.

16. Briskin DP. Medicinal plants and phytomedicines. Linking plant biochemistry and physiology to human health. Plant Physiol. 2000;124(2):507-14.

17. Park WH, Lee SK, Kim CH. A Korean herbal medicine, Panax notoginseng, prevents liver fibrosis and hepatic microvascular dysfunction in rats. Life Sci. 2005;76(15):1675-90.

18. Ng TB. Pharmacological activity of sanchi ginseng (Panax notoginseng). J Pharm Pharmacol. 2006:58(8):1007-19.

19. Niu Y, Luo H, Sun C, Yang TJ, Dong L, Huang L, et al. Expression profiling of the triterpene saponin biosynthesis genes FPS, SS, SE, and DS in the medicinal plant Panax notoginseng. Gene. 2014;533(1):295-303.

20. Xia P, Guo H, Liang Z, Cui X, Liu Y, Liu F. Nutritional composition of sanchi (Panax notoginseng) seed and its potential for industrial use. Genet Resour Crop Ev. 2014;61(3):663-7.

21. Cui XM, Huang LQ, Guo LP, Liu DH. Chinese Sanqi industry status and development countermeasures. Zhongguo Zhong Yao Za Zhi. 2014;39(4): 553-7.

22. Feller A, Machemer K, Braun EL, Grotewold E. Evolutionary and comparative analysis of MYB and bHLH plant transcription factors. Plant J. 2011;66(1):94-116.

23. Bakshi M, Oelmüller R. WRKY transcription factors: Jack of many trades in plants. Plant Signal Behav. 2014;9(2):e27700.

24. Li SB, Xie ZZ, Hu CG, Zhang JZ. A review of auxin response factors (ARFs) in plants. Front Plant Sci. 2016;7:47.

25. Gu C, Guo ZH, Hao PP, Wang GM, Jin ZM, Zhang SL. Multiple regulatory roles of AP2 /ERF transcription factor in angiosperm. Bot Stud. 2017;58(1):6.

26. Mathew IE, Agarwal P. May the fittest protein evolve: favoring the plantspecific origin and expansion of NAC transcription factors. BioEssays. 2018; 40(8):e1800018.

27. Li QF, Lu J, Yu JW, Zhang CQ, He JX, Liu QQ. The brassinosteroid-regulated transcription factors BZR1/BES1 function as a coordinator in multisignalregulated plant growth. Biochim Biophys Acta Gene Regul Mech. 2018; 1861(6):561-71.

28. Lyu T, Cao J. Cys $2 / H i s_{2}$ zinc-finger proteins in transcriptional regulation of flower development. Int J Mol Sci. 2018;19(9).

29. Jung JK, McCouch S. Getting to the roots of it: genetic and hormonal control of root architecture. Front Plant Sci. 2013;4:186.

30. Ljung K. Auxin metabolism and homeostasis during plant development. Development. 2013;140(5):943-50.

31. Cosgrove DJ. Growth of the plant cell wall. Nat Rev Mol Cell Biol. 2005;6(11): 850-61.

32. Paque S, Mouille G, Grandont L, Alabadí D, Gaertner C, Goyallon A, et al. AUXIN BINDING PROTEIN1 links cell wall remodeling, auxin signaling, and cell expansion in Arabidopsis. Plant Cell. 2014;26(1):280-95.

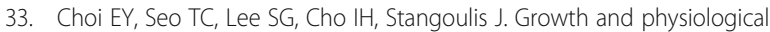
responses of Chinese cabbage and radish to long-term exposure to elevated carbon dioxide and temperature. Hort Environ Biotechnol. 2011; 52(4):376-86.

34. Ursache R, Nieminen K, Helariutta Y. Genetic and hormonal regulation of cambial development. Physiol Plant. 2013;147(1):36-45.

35. Matsuo T, Yoneda T, Itoo S. Identification of free cytokinins and the changes in endogenous levels during tuber development of sweet potato (Ipomoea batatas Lam.). Plant Cell Physiol. 1983:24(7):1305-12.

36. Nakatani M, Komeichi M. Changes in endogenous indole acetic acid level during development of roots in sweet potato. Japan J Crop Sci. 1992;61(4): 683-4.

37. Abelenda JA, Navarro C, Prat S. From the model to the crop: genes controlling tuber formation in potato. Curr Opin Biotechnol. 2011;22(2): 287-92.

38. Carrera E, Bou J, Garcia-Martinez JL, Prat S. Changes in GA20-oxidase gene expression strongly affect stem length, tuber induction and tuber yield of potato plants. Plant J. 2000;22(3):247-56.

39. Muñiz García MN, Muro MC, Mazzocchi LC, País SM, Stritzler M, Schlesinger $M$, et al. The protein phosphatase 2A catalytic subunit StPP2Ac2b acts as a positive regulator of tuberization induction in Solanum tuberosum L. Plant Mol Biol. 2017:93(3):227-45.

40. Xu X, van-Lammeren AA, Vermeer $E$, Vreugdenhil D. The role of gibberellin, abscisic acid, and sucrose in the regulation of potato tuber formation in vitro. Plant Physiol. 1998;117(2):575-84. 
41. El-Antably HM, Wareing PF, Hillman J. Some physiological responses to d,I abscisin (dormin). Planta. 1967;73(1):74-90.

42. Vreugdenhil D, Bindels $P$, Reinhoud $P$, Klocek J, Hendriks T. Use of the growth retardant tetcyclacis for potato-tuber formation in vitro. Plant Growth Regul. 1994;14(3):257-65.

43. Seo M, Hanada A, Kuwahara A, Endo A, Okamoto M, Yamauchi Y, et al. Regulation of hormone metabolism in Arabidopsis seeds: phytochrome regulation of abscisic acid metabolism and abscisic acid regulation of gibberellin metabolism. Plant J. 2006;48(3):354-66.

44. Oh E, Yamaguchi S, Hu J, Yusuke J, Jung B, Paik I, et al. PIL5, a phytochrome-interacting bHLH protein, regulates gibberellin responsiveness by binding directly to the GAI and RGA promoters in Arabidopsis seeds. Plant Cell. 2007:19(4):1192-208.

45. Kong X, Tian H, Yu Q, Zhang F, Wang R, Gao S, et al. PHB3 maintains root stem cell niche identity through ROS-responsive AP2/ERF transcription factors in Arabidopsis. Cell Rep. 2018;22(5):1350-63.

46. Cai XT, Xu P, Zhao PX, Liu R, Yu LH, Xiang CB. Arabidopsis ERF109 mediates cross-talk between jasmonic acid and auxin biosynthesis during lateral root formation. Nat Commun. 2014;5:5833.

47. Zhou W, Lozano-Torres JL, Blilou I, Zhang X, Zhai Q, Smant G, et al. A jasmonate signaling network activates root stem cells and promotes regeneration. Cell. 2019;177(4):942-56.

48. Mitsuda N, Seki M, Shinozaki K, Ohme-Takagi M. The NAC transcription factors NST1 and NST2 of Arabidopsis regulate secondary wall thickenings and are required for anther dehiscence. Plant Cell. 2005;17(11):2993-3006.

49. Zhong $\mathrm{RQ}$, Lee $\mathrm{CH}$, McCarthy $\mathrm{RL}$, Reeves $\mathrm{CK}$, Jones $\mathrm{EG}$, Ye ZH. Transcriptional activation of secondary wall biosynthesis by rice and maize NAC and MYB transcription factors. Plant Cell Physiol. 2011;52(10):1856-71.

50. Ohashi-Ito K, Oda Y, Fukuda H. Arabidopsis VASCULAR-RELATED NACDOMAIN6 directly regulates the genes that govern programmed cell death and secondary wall formation during xylem differentiation. Plant Cell. 2010; 22(10):3461-73.

51. Han Q, Zhang J, Li H, Luo Z, Ziaf K, Ouyang B, et al. Identification and expression pattern of one stress-responsive NAC gene from Solanum lycopersicum. Mol Bio Rep. 2012;39(2):1713-20.

52. Sablowski RW, Meyerowitz EM. A homolog of NO APICAL MERISTEM is an immediate target of the floral homeotic genes APETALA3/PISTILLATA. Cell. 1998;92(1):93-103.

53. Ingram P, Dettmer J, Helariutta Y, Malamy JE. Arabidopsis lateral root development 3 is essential for early phloem development and function, and hence for normal root system development. Plant J. 2011;68(3):455-67.

54. Peng Y, Ma W, Chen L, Yang L, Li S, Zhao H, et al. Control of root meristem size by DAI-RELATED PROTEIN2 in Arabidopsis. Plant Physiol. 2013;161:1542-56.

55. Koch K. Sucrose metabolism: regulatory mechanisms and pivotal roles in sugar sensing and plant development. Curr Opin Plant Biol. 2004;7(3):235-46.

56. Usuda H, Demura T, Shimogawara K, Fukuda H. Development of sink capacity of the "storage root" in a radish cultivar with a high ratio of "storage root" to shoot. Plant Cell Physiol. 1999;40(4):369-77.

57. Yang M, Zhu LP, Pan C, Xu L, Liu Y, Ke W, et al. Transcriptomic analysis of the regulation of rhizome formation in temperate and tropical lotus (Nelumbo nucifera). Sci Rep. 2015;5:13059.

58. Jackson SD. Multiple signaling pathways control tuber induction in potato. Plant Physiol. 1999;119(1):1-8.

59. Zrenner R, Salanoubat M, Willmitzer L, Sonnewald U. Evidence of the crucial role of sucrose synthase for sink strength using transgenic potato plants (Solanum tuberosum L.). Plant J. 1995;7(1):97-107.

60. Shewry PR. Tuber storage proteins. Ann Bot. 2003:91(7):755-69.

61. Hong YF, Liu CY, Cheng KJ, Hour AL, Chan MT, Tseng TH, et al. The sweet potato sporamin promoter confers high-level phytase expression and improves organic phosphorus acquisition and tuber yield of transgenic potato. Plant Mol Biol. 2008;67(4):347-61.

62. Liu L, Huang Y, Huang X, Yang J, Wu W, Xu Y, et al. Characterization of the dioscorin gene family in Dioscorea alata reveals a role in tuber development and environmental response. Int J Mol Sci. 2017;18(7):1579.

63. Kim SI, Kweon SM, Kim EA, Kim JY, Kim S, Yoo JS, et al. Characterization of RNase-like major storage protein from the ginseng root by proteomic approach. J Plant Physiol. 2004;161(7):837-45.

64. Liu J, Luo X, Shaff J, Liang C, Jia X, Li Z, et al. A promoter-swap strategy between the AtALMT and AtMATE genes increased Arabidopsis aluminum resistance and improved carbon-use efficiency for aluminum resistance. Plant J. 2012;71(2):327-37.
65. Balzergue C, Dartevelle T, Godon C, Laugier E, Meisrimler C, Teulon JM, et al. Low phosphate activates STOP1-ALMT1 to rapidly inhibit root cell elongation. Nat Commun. 2017;8:15300.

66. Xia J, Yamaji N, Che J, Shen RF, Ma JF. Normal root elongation requires arginine produced by argininosuccinate lyase in rice. Plant J. 2014;78(2): 215-26.

67. Van Etten $\mathrm{CH}$, Miller RW, Wolff IA, Jones Q. Nutrients in seeds, amino acid composition of seeds from 200 angiospermous plant species. J Agric Food Chem. 1963;11(5):399-410.

68. Canene-Adams K. Preparation of formalin-fixed paraffin-embedded tissue for immunohisto chemistry. Methods Enzymol. 2013;533:225-33.

69. Li R, Yu C, Li Y, Lam TW, Yiu SM, Kristiansen K, et al. SOAP2:an improved ultrafast tool for short read alignment. Bioinformatics. 2009;25(15):1966-7.

70. Mortazavi A, Williams BA, McCue K, Schaeffer L, Wold B. Mapping and quantifying mammalian transcriptomes by RNA-Seq. Nat Methods. 2008; 5(7):621-8.

\section{Publisher's Note}

Springer Nature remains neutral with regard to jurisdictional claims in published maps and institutional affiliations.
Ready to submit your research? Choose BMC and benefit from:

- fast, convenient online submission

- thorough peer review by experienced researchers in your field

- rapid publication on acceptance

- support for research data, including large and complex data types

- gold Open Access which fosters wider collaboration and increased citations

- maximum visibility for your research: over $100 \mathrm{M}$ website views per year

At $\mathrm{BMC}$, research is always in progress.

Learn more biomedcentral.com/submissions 